\title{
Exploiting Linearity of Modular Multiplication
}

\author{
Hamdi Murat Yıldırım( $\left.{ }^{(}\right)$ \\ Department of Computer Technology and Information Systems, \\ Bilkent University, 06800 Ankara, Turkey \\ hmurat@bilkent.edu.tr
}

\begin{abstract}
The XOR $\bigoplus$ and the addition $\boxplus$ operations have been widely used as building blocks for many cryptographic primitives. These operations and the multiplication $\odot$ operation are successively used in the design of IDEA and the MESH block ciphers. This work presents several interesting algebraic properties of the multiplication operation. By fixing one operand, we obtain vector valued function $\boldsymbol{g}_{Z}$ on $\mathbb{Z}_{2}^{n}$, associated with $\odot$. In this paper we show that the nonlinearity of $\boldsymbol{g}_{Z}$ remains the same under some transformations of $Z$ and moreover we give an upper bound for the nonlinearity of $\boldsymbol{g}_{Z}$ when $Z$ is a power of 2 . Under weak-key assumptions, we furthermore present a list of new linear relations for 1round IDEA cipher, some of directly derived and others algorithmically generated using these relations and known ones. We extend the largest linear weak key class for IDEA cipher with size $2^{23}$ to derive such a class with sizes $2^{24}$. Under the independent key subblocks (subkeys) and weak-key assumptions we derive many linear relations for IDEA cipher using linear relations for 1-round IDEA cipher.
\end{abstract}

Keywords: IDEA cipher - Nonlinearity - Modular multiplication · Boolean functions · Cryptanalysis

\section{Introduction}

Block ciphers can be used to build other cryptographic primitives such as stream ciphers, hash functions, message authentication codes and cryptographically secure pseudorandom number generators. Both block ciphers and stream ciphers provide confidentiality, which ensures that information is accessible only to those authorized for access, one of the goals of information security. The addition modulo $2^{n}(\boxplus)$ and exclusive-OR (XOR) $(\bigoplus$, bitwise addition on modulo 2$)$ are operations and have been widely used as building blocks in many cryptosystems: in RC6, Twofish, MARS, FEAL, SAFER as block ciphers and in ChaCha, Phelix, Snow as stream ciphers. The design of both the International Data Encryption Algorithm (IDEA) [4], the MESH block ciphers [9], WIDEA [3] cipher and RIDEA cipher [12] are based on the successive use of these operations and the multiplication modulo $2^{16}+1(\odot)$ operation. Extensive survey of such

(C) Springer Nature Switzerland AG 2020

D. Slamanig et al. (Eds.): MACIS 2019, LNCS 11989, pp. 249-269, 2020.

https://doi.org/10.1007/978-3-030-43120-4_19 
block ciphers whose design following the Lai-Massey design paradigm and their analyses are provided by Nakahara [8]. IDEA was used in Pretty Good Privacy (PGP), which is a widely used computer program that provides confidentiality, authentication and data integrity. There are other applications of multiplication modulo $2^{16}+1(\odot)$, which are encountered in residue number systems and Fermat number transform and studies about improving its efficiency $[1,6]$ Some algebraic properties of the operations $\boxplus, \odot$ and $\oplus$ have already been exploited to cryptanalyze the first 2-round of IDEA in [5]. 15 linear relations for 1-round IDEA cipher, which are derived by considering the linearity of both XOR $\oplus$ and the addition $\boxplus$ operation and also linearity of the multiplication $\odot$ for values 0 and 1 , are used to derive the linear weak key class for IDEA cipher with size $2^{23}$ [2]. In this respect, nonlinearity is one of the well-known criterion for evaluating cryptographic Boolean functions. Note that the nonlinearity of both addition and multiplication is considered as high because of their polynomial expressions according to Theorem 3 and 4 in [4]. This is one of the reasons they are used in IDEA cipher. On the other hand, we consider the widely known and accepted measurement for nonlinearity based on the Hamming distance presented in [10] to study the nonlinearity of the multiplication operation. It is proved that this type of nonlinearity of $\odot$ is zero for six cases for $n \geq 2$ [12].

\subsection{Contribution}

In this paper we view each operation of IDEA cipher as a vector valued boolean function from $\mathbb{Z}_{2}^{n} \times \mathbb{Z}_{2}^{n}$ to $\mathbb{Z}_{2}^{n}$. Note that the designer of IDEA cipher just considers the case $n=16$. We fix one operand of each operation to have a vector valued function from $\mathbb{Z}_{2}^{n}$ to $\mathbb{Z}_{2}^{n}$ and we use the nonlinearity measurement in [10]. We give an upper bound for its nonlinearity when $Z=2^{k}, 2 \leq k \leq\lceil(n-1) / 2\rceil$. This means that the nonlinearity of the operation $\odot$ is low for small values of $k$. In fact, it is expected that the nonlinearity of such building blocks of block ciphers should be high. In Sect. 3 for the operation $\odot$, we construct a family of transformations that leaves nonlinearity invariant. In Sect.4, in addition to 15 linear relations holding with probability one for 1-round IDEA cipher given in [2], we use all cases making nonlinearity of IDEA cipher's operations zero in order to derive such extra 39 linear relations. Moreover, we devise an algorithm to derive 201 more such linear relation considering these 54 relations. Section 5 presents one linear weak key class for IDEA cipher with size $2^{24}$, which is extended from a largest linear weak key class for IDEA cipher with size $2^{23}$ presented in [2] and a method for 438 linear relations for IDEA cipher considering subkeys chosen independently and 255 linear relations for 1-round IDEA cipher.

\section{Preliminaries}

We shall use the following notations throughout the rest of the paper:

- $x \oplus y=x+y(\bmod 2)$ for $x, y \in \mathbb{Z}_{2}$;

- $\mathbb{Z}_{2}^{n}=\mathbb{Z}_{2} \times \ldots \times \mathbb{Z}_{2}$ (n-times) denotes the $n$-dimensional vector space over $\mathbb{Z}_{2}$; 
- When $\boldsymbol{A}=\left(a_{n}, a_{n-1}, \ldots, a_{1}\right)$ and $\boldsymbol{X}=\left(x_{n}, x_{n-1}, \ldots, x_{1}\right) \in \mathbb{Z}_{2}^{n}$,

- $\boldsymbol{A} \oplus \boldsymbol{X}=\left(a_{n} \oplus x_{n}, a_{n-1} \oplus+x_{n-1}, \ldots, a_{1} \oplus x_{1}\right)$.

- the dot product $\boldsymbol{A} \cdot \boldsymbol{X}=\left(\sum_{i=1}^{n} a_{i} x_{i}\right)(\bmod 2)=a_{n} x_{n} \oplus a_{n-1} x_{n-1} \oplus$ $\ldots \oplus a_{1} x_{1}$.

- for $\lambda \in \mathbb{Z}_{2}, l_{\boldsymbol{A}, \lambda}: \mathbb{Z}_{2}^{n} \rightarrow \mathbb{Z}_{2}$ be the function defined by

$l_{\boldsymbol{A}, \lambda}(\boldsymbol{X})=\boldsymbol{A} \cdot \boldsymbol{X} \oplus \lambda$ is called an affine function (respectively linear) if $\lambda \neq 0$ (respectively $\lambda=0$ ).

- $\mathcal{A}=\left\{l_{\boldsymbol{A}, \lambda} \mid \boldsymbol{A} \in \mathbb{Z}_{2}^{n}, \lambda \in \mathbb{Z}_{2}\right\}$ denotes the set of all affine functions on $\mathbb{Z}_{2}^{n}$.

- $|S|$ denotes the cardinality of the set $S$.

It is easy to introduce the addition $\boxplus$, the multiplication $\odot$ and $\mathrm{XOR} \oplus$ operations for any positive integer $n$ as functions from $\mathbb{Z}_{2}^{n} \times \mathbb{Z}_{2}^{n} \rightarrow \mathbb{Z}_{2}^{n}=\mathbb{Z}_{2} \times \ldots \times \mathbb{Z}_{2}$ (n-times) as follows:

Let $\mathbb{Z}_{2^{n}}=\left\{0,1, \ldots, 2^{n}-1\right\}, \mathbb{Z}_{2^{n}+1}^{*}=\left\{1,2, \ldots, 2^{n}\right\}$, and let $v: \mathbb{Z}_{2^{n}} \rightarrow \mathbb{Z}_{2}^{n}$ be a function defined by $v(X)=\boldsymbol{X}$,

where $\boldsymbol{X}=\left(x_{n}, \ldots, x_{2}, x_{1}\right)$ is a bit representation of $X=\sum_{i=1}^{n} x_{i} 2^{i-1} \in \mathbb{Z}_{2^{n}}$ and

$d: \mathbb{Z}_{2^{n}+1}^{*} \rightarrow \mathbb{Z}_{2^{n}}$ be a function defined by $d(X)=X$ if $X \neq 2^{n}$ and $d\left(2^{n}\right)=0$.

With this convention, the addition $\left(\bmod 2^{n}\right)$, $\boxplus$, the multiplication, $\odot$, $\left(\bmod 2^{n}+1\right)$ and the XOR $\oplus$ operations produce the three functions $\boldsymbol{f}, \boldsymbol{g}$ and $\boldsymbol{h}: \mathbb{Z}_{2}^{n} \times \mathbb{Z}_{2}^{n} \rightarrow \mathbb{Z}_{2}^{n}$ :

The addition operation $\boxplus ; \boldsymbol{f}(\boldsymbol{X}, \boldsymbol{Z})=\boldsymbol{X} \boxplus \boldsymbol{Z}=v\left(X+Z \quad\left(\bmod 2^{n}\right)\right)$.

The multiplication operation $\odot ; \boldsymbol{g}(\boldsymbol{X}, \boldsymbol{Z})=\boldsymbol{X} \odot \boldsymbol{Z}=v\left(d\left(d^{-1}(X) d^{-1}(Z)\right.\right.$

$\left.\left.\left(\bmod 2^{n}+1\right)\right)\right)$, where $d^{-1}$ is the inverse $d$.

The XOR operation $\bigoplus ; \boldsymbol{h}(\boldsymbol{X}, \boldsymbol{Z})=\boldsymbol{X} \oplus \boldsymbol{Z}=\left(x_{n} \oplus z_{n}, x_{n-1} \oplus z_{n-1}, \ldots\right.$, $\left.x_{1} \oplus z_{1}\right)$.

Notation: for any $Z \in \mathbb{Z}_{2^{n}}, v(Z)=\boldsymbol{Z} \in \mathbb{Z}_{2}^{n}$, we denote by $\boldsymbol{f}_{Z}, \boldsymbol{g}_{Z}$ and $\boldsymbol{h}_{Z}$ the following vector valued functions $\mathbb{Z}_{2}^{n} \rightarrow \mathbb{Z}_{2}^{n}: \boldsymbol{f}_{Z}(\boldsymbol{X})=f(\boldsymbol{X}, \boldsymbol{Z}), \boldsymbol{g}_{Z}(\boldsymbol{X})=$ $g(\boldsymbol{X}, \boldsymbol{Z})$ and $\boldsymbol{h}_{Z}(\boldsymbol{X})=h(\boldsymbol{X}, \boldsymbol{Z})$.

Let $f: \mathbb{Z}_{2}^{n} \rightarrow \mathbb{Z}_{2}$ be any function and let $H(f)$ denote the Hamming distance from $f$ to the set of all affine functions $\mathcal{A}$ on $\mathbb{Z}_{2}^{n}$. Namely,

$$
H(f)=\min \left\{E_{\boldsymbol{A}, \lambda}(f) \mid \boldsymbol{A} \in \mathbb{Z}_{2}^{n}, \lambda \in \mathbb{Z}_{2}\right\}
$$

where $E_{\boldsymbol{A}, \lambda}(f)=\left|\left\{\boldsymbol{X} \in \mathbb{Z}_{2}^{n} \mid f(\boldsymbol{X}) \neq l_{\boldsymbol{A}, \lambda}(\boldsymbol{X})=\boldsymbol{A} \cdot \boldsymbol{X} \oplus \lambda\right\}\right|$.

This non-negative integer $H(f)$ attached to $f: \mathbb{Z}_{2}^{n} \rightarrow \mathbb{Z}_{2}$ is called the nonlinearity of $f$.

It is clear that $H(f)=0$ iff $f$ is an affine function. The concept of nonlinearity of arbitrarily vector function $\boldsymbol{F}: \mathbb{Z}_{2}^{n} \rightarrow \mathbb{Z}_{2}^{k}$ was introduced in [10] as follows: Let $\boldsymbol{F}=\left(f_{k}, \ldots, f_{1}\right), f_{i}: \mathbb{Z}_{2}^{n} \rightarrow \mathbb{Z}_{2}$, where $1 \leq i \leq k$.

\section{Definition 1.}

$$
N(\boldsymbol{F})=\min _{\boldsymbol{C}=\left(c_{1}, \ldots, c_{k}\right) \in \mathbb{Z}_{2}^{k} \backslash\{0\}}\left\{H\left(\boldsymbol{C} \cdot \boldsymbol{F}=c_{k} f_{k} \oplus c_{k-1} f_{k-1} \oplus \ldots \oplus c_{1} f_{1}\right)\right\}
$$

Definition 2. Let $f$ be a function from $\mathbb{Z}_{2}^{n}$ to $\mathbb{Z}_{2}$. The truth table of $f$ is an ordered $2^{n}$-tuple $\left(f(\mathbf{0}), f(\mathbf{1}), \ldots, f\left(\mathbf{2}^{n}-\mathbf{1}\right)\right) \in \mathbb{Z}_{2}^{2^{n}}$, which is denoted by $T_{f}$. 


\section{Nonlinearity of Multiplication Operation}

It is a well-known fact that for every $Z \in \mathbb{Z}_{2^{n}}$, the nonlinearity $N\left(\boldsymbol{f}_{Z}\right)$ and $N\left(\boldsymbol{h}_{Z}\right)$ of $\boldsymbol{f}_{Z}$ and $\boldsymbol{h}_{Z}$ are equal to 0 . However, the nonlinearity $N\left(\boldsymbol{g}_{Z}\right)$ of the vector function $\boldsymbol{g}_{Z}$ is not zero for every $Z \in \mathbb{Z}_{2^{n}}$. The following theorem, which is proved in [12], gives a list of $Z$ values such that $N\left(\boldsymbol{g}_{Z}\right)$ is zero.

Theorem 1. For $n \geq 2$, the nonlinearity $N\left(\boldsymbol{g}_{Z}\right)$ of the vector function $\boldsymbol{g}_{Z}(\boldsymbol{X})=$ $\boldsymbol{g}(\boldsymbol{X}, \boldsymbol{Z})$ is zero for $Z=0,1,2,2^{n-1}, 2^{n-1}+1,2^{n}-1$.

Remark 1. When $n \leq 12$, we checked that the values of $Z$ in Theorem 1 were the only ones for which $N\left(\boldsymbol{g}_{Z}\right)=0$. It is an open problem whether this is the case for $n>12$.

Using the following proposition, it is enough to calculate $N\left(\boldsymbol{g}_{Z}\right)$ for given $Z$ value to determine one, two or three related values for the vector function of the multiplication operation having the same nonlinearity.

\section{Proposition 1}

(1) For $n \in \mathbb{Z}_{+}$such that $\operatorname{gcd}\left(A, 2^{n}+1\right)=1$, we have $N\left(\boldsymbol{g}_{A}\right)=N\left(\boldsymbol{g}_{B}\right)$ when $A B \equiv 1\left(\bmod 2^{n}+1\right)$.

(2) $N\left(\boldsymbol{g}_{A}\right)=N\left(\boldsymbol{g}_{B}\right)$ when $A+B \equiv 0\left(\bmod 2^{n}+1\right)$.

(3) $N\left(\boldsymbol{g}_{2^{k}}\right)=N\left(\boldsymbol{g}_{2^{s}}\right)$ when $k+s=n$ for $k, s \geq 0$.

Proof. For part 1 , we have $\boldsymbol{g}_{B}(\boldsymbol{X})=\boldsymbol{g}_{A^{-1}}(\boldsymbol{X})$ since $A B \equiv 1\left(\bmod 2^{n}+1\right)$. $N\left(\boldsymbol{g}_{A}\right)=N\left(\left(\boldsymbol{g}_{A}\right)^{-1}\right)=N\left(\boldsymbol{g}_{B}\right)$ follows from Theorem 1 in [10].

For part 2, the case $A=B=0$ is trivial. For other $(A, B)$ pairs, one can use the obvious relation $v^{-1}\left(\boldsymbol{g}_{A}(\boldsymbol{X})\right)+v^{-1}\left(\boldsymbol{g}_{B}(\boldsymbol{X})\right) \equiv 0\left(\bmod 2^{n}+1\right)$ to complete the proof of this part.

For part 3 , for $k+s=n$, we obtain that $2^{s}\left(2^{k}+2\left(2^{s}\right)^{-1}\right) \equiv 2^{n}+2 \equiv 1$ $\left(\bmod 2^{n}+1\right)$. Here $\left(2^{s}\right)^{-1} \equiv 2^{k}+2\left(2^{s}\right)^{-1}\left(\bmod 2^{n}+1\right)$ and we have $\left(2^{s}\right)^{-1}+2^{k} \equiv$ $0\left(\bmod 2^{n}+1\right)$. By part 2, we get $N\left(\boldsymbol{g}_{\left(2^{s}\right)^{-1}}\right)=N\left(\boldsymbol{g}_{2^{k}}\right)$. From Theorem 1 in [10], we know that $N\left(\boldsymbol{g}_{\left(2^{s}\right)^{-1}}\right)=N\left(\boldsymbol{g}_{2^{s}}\right)$. This completes the proof.

Since there is no efficient algorithm to compute $N\left(\boldsymbol{g}_{Z}\right)$ in general, we can look for an upper bound for some values of $Z$. The following theorem gives a partial solution to the problem:

Theorem 2. For $n \geq 3$ and $2 \leq k \leq\lceil(n-1) / 2\rceil$, we have $N\left(\boldsymbol{g}_{Z}\right) \leq 2^{k-1}$ when

(i) $Z=2^{k}$ and $Z=2^{n-k}$.

(ii) $Z+2^{k} \equiv 0 \quad\left(\bmod 2^{n}+1\right)$.

(iii) $Z 2^{k} \equiv 1 \quad\left(\bmod 2^{n}+1\right)$.

Proof. Assume that $n \geq 3$ and $2 \leq k \leq\lceil(n-1) / 2\rceil$. For every $\boldsymbol{X} \in \mathbb{Z}_{2}^{n}$,

let $\boldsymbol{g}_{2^{k}}(\boldsymbol{X})=\left(\boldsymbol{g}_{2^{k}}{ }^{(n)}(\boldsymbol{X}), \ldots, \boldsymbol{g}_{2^{k}}{ }^{(2)}(\boldsymbol{X}), \boldsymbol{g}_{2^{k}}{ }^{(1)}(\boldsymbol{X})\right)$, and $\boldsymbol{g}_{2^{k}}{ }^{(i)}(\boldsymbol{X})$ be $i^{\text {th }}$ coordinate function of $\boldsymbol{g}_{2^{k}}(\boldsymbol{X})$. 
Since $\boldsymbol{g}_{2}(0)=2^{n}-1, \boldsymbol{g}_{2}\left(2^{n-1}\right)=0$ and $\boldsymbol{g}_{2}(2 j)$ is even and $\boldsymbol{g}_{2}(2 j+1)$ is odd for all $j \in\left\{1, \ldots, 2^{n-1}-1\right\}$, the truth table of $\boldsymbol{g}_{2}{ }^{(1)}, T_{\boldsymbol{g}_{2}(1)}=S^{2^{n}}$, where $S^{2^{n}}=$ $\left(s_{2^{n}}, \ldots, s_{1}\right)=(1,0, \ldots, 0,0,1, \ldots, 1) \in \mathbb{Z}_{2}^{2^{n}}, s_{2^{n}}=1, s_{2^{n-1}}=0, s_{2^{n-1}+m}=0$ and $s_{2^{n-1}-m}=1$ for all $m \in\left\{1, \ldots, 2^{n-1}-1\right\}$. Then the truth table of $T_{\boldsymbol{g}_{2^{k}}}$ becomes $(\underbrace{S^{2^{n-k+1}}, \ldots, S^{2^{n-k+1}}}_{\left(2^{k-1}\right)-\text { times }})$. Therefore, $\boldsymbol{g}_{2}^{(1)}(\boldsymbol{X})=\overline{x_{1}} \overline{x_{2}} \ldots \overline{x_{n-1}} \oplus x_{n}$ and

$\boldsymbol{g}_{2^{k}}^{(1)}(\boldsymbol{X})=\overline{x_{1}} \overline{x_{2}} \ldots \overline{x_{n-k}} \oplus x_{n-k+1}$ according to their truth tables, where $\overline{x_{i}}=$ $x_{i} \oplus 1$. We know that $\boldsymbol{g}_{2^{k}}^{(1)}(\boldsymbol{X}) \oplus \boldsymbol{g}_{2^{k}}^{(2)}(\boldsymbol{X})=\boldsymbol{g}_{2^{k-1}}^{(1)}(\boldsymbol{X})$ since by the proof of Theorem 1, $y_{2} \oplus y_{1}=x_{1}$ for $\boldsymbol{g}_{2}(\boldsymbol{X})=\boldsymbol{Y}$. The hamming distance between $\boldsymbol{g}_{2^{k}}^{(1)}(\boldsymbol{X})$ and $x_{n-k+1}$ is $2^{k}$.

This implies that $N\left(\boldsymbol{g}_{2^{k}}^{(1)}(\boldsymbol{X})\right) \leq 2^{k}$. By Theorem 12 in [13], $2^{k} \leq$ $N\left(\boldsymbol{g}_{2^{k}}^{(1)}(\boldsymbol{X})\right)$ since the term $x_{1} \ldots x_{n-k}$ is not properly covered (see Definition 9 in [13]) by any other terms in $\boldsymbol{g}_{2^{k}}^{(1)}(\boldsymbol{X})$. Then, $N\left(\boldsymbol{g}_{2^{k}}{ }^{(1)}(\boldsymbol{X})\right)=2^{k}$ and we get $N\left(\boldsymbol{g}_{2^{k}}{ }^{(1)}(\boldsymbol{X}) \oplus \boldsymbol{g}_{2^{k}}{ }^{(2)}(\boldsymbol{X})\right)=N\left(\boldsymbol{g}_{2^{k-1}}{ }^{(1)}(\boldsymbol{X})\right)=2^{k-1}$. Hence, $N\left(\boldsymbol{g}_{2^{k}}(\boldsymbol{X})\right) \leq$ $2^{k-1}$ by using Definition 1 .

The remaining parts of this theorem can be easily proven by Proposition 1 .

Remark 2. When $n \leq 16$, we checked that the upper bound was tight, namely $N\left(\boldsymbol{g}_{Z}\right)=2^{k-1}$, for the choices of $Z$ above. It is an open problem whether this is the case when $n>16$.

\section{Linear Relations for 1-Round IDEA}

\subsection{Linear Relations for Operations}

For a fixed operation $\bowtie \in\{\boxplus, \odot, \oplus\}$ and $z \in \mathbb{Z}_{2^{n}}$, we consider mapping $\mathbb{Z}_{2}^{n} \rightarrow \mathbb{Z}_{2}^{n}$ defined by $\mathbf{X} \rightarrow \mathbf{X} \bowtie \mathbf{Z}=\mathbf{Y}(\mathbf{Z}=v(z))$.

We have discussed the nonlinearity of this vector valued multiplication function for some special cases. When $\bowtie$ is the XOR operation $\bigoplus$, it is clear that the dot product is distributive over $\bigoplus$, and therefore we get $\mathbf{A} \cdot(\mathbf{X} \oplus \mathbf{Z})=$ $\mathbf{A} \cdot \mathbf{X} \oplus \mathbf{A} \cdot \mathbf{Z}=\mathbf{A} \cdot \mathbf{Y}$, or equivalently

$$
\mathbf{A} \cdot \mathbf{X} \oplus \mathbf{A} \cdot \mathbf{Y} \oplus \mathbf{A} \cdot \mathbf{Z}=0 \text { for every } \mathbf{A} \in \mathbb{Z}_{2}^{n}
$$

Similarly for $\bowtie=\boxplus$, it is easy to see that $\mathbf{1} \cdot(\mathbf{X} \boxplus \mathbf{Z})=\mathbf{1} \cdot \mathbf{X} \oplus \mathbf{1} \cdot \mathbf{Z}=\mathbf{1} \cdot \mathbf{Y}$, or equivalently

$$
\mathbf{1} \cdot \mathbf{X} \oplus \mathbf{1} \cdot \mathbf{Y} \oplus \mathbf{1} \cdot \mathbf{Z}=0
$$

So for $\mathbf{X} \bowtie \mathbf{Z}=\mathbf{Y}$ it makes sense to search relations in the form

$$
\mathbf{A} \cdot \mathbf{X} \oplus \mathbf{B} \cdot \mathbf{Y} \oplus \mathbf{C} \cdot \mathbf{Z} \oplus \lambda=0 \text { for some } \mathbf{A}, \mathbf{B}, \mathbf{C} \in \mathbb{Z}_{2}^{n} \text { and } \lambda \in \mathbb{Z}_{2} \text {. }
$$

As it can be seen from the proof of Theorem 1 [12], we get the following linear relations for every $\mathbf{X}=v(x) \in \mathbb{Z}_{2}^{n}$ such that $\mathbf{X} \odot \mathbf{Z}=\mathbf{Y}$ :

$$
\mathbf{1} \cdot \mathbf{X} \oplus \mathbf{1} \cdot \mathbf{Y} \oplus \mathbf{1} \cdot \mathbf{Z} \oplus 1=0 \text { for } z \in\{0,1\}
$$




$$
\begin{aligned}
\mathbf{3} \cdot \mathbf{X} \oplus \mathbf{1} \cdot \mathbf{Y} \oplus \mathbf{1} \cdot \mathbf{Z} \oplus 1=0 \text { for } z \in\left\{2^{n-1}, 2^{n-1}+1\right\} \\
\mathbf{1} \cdot \mathbf{X} \oplus \mathbf{3} \cdot \mathbf{Y} \oplus \mathbf{1} \cdot \mathbf{Z}=0 \text { for } z \in\left\{2,2^{n}-1\right\},
\end{aligned}
$$

where $v(z)=\mathbf{Z}$.

\subsection{A New List of Linear Relations}

For 1-round IDEA, 15 linear relations hold with probability one are derived due to the linearity of operations of IDEA (see equations in 1,2,4) in paper [2]. These relations marked by $(*)$ are given in Table 1 . Note that for each round of IDEA, four of the six 16-bit key subblocks $\mathbf{Z}_{i}$ 's $(i=\{1,4,5,6\})$ are involved by the multiplication operation $\odot$. In order to derive each of these linear relation, at least one of those key subblocks were restricted to take 0 and 1 (see Example 1 and Table 1). Additional key values, $2,2^{n}-1,2^{n-1}$ and $2^{n-1}+1$, making the nonlinearity of the vector valued function $\mathbf{g}_{z}$ of $\odot$ zero were discovered in [12]. Similar to the work in paper [2], we take into account 0,1 or these key values as round multiplicative keys to derive extra 39 linear relations, which are not marked by $\left(^{*}\right)$ in Table 1 . All these 54 linear relations (holding with probability one) with the related key subblocks restrictions are listed in Table 1. Notice that each linear relation for 1-round IDEA should be based on linear relations for the operations used in IDEA cipher. Hence under some round key subblocks restrictions (weak key assumptions), we can express a linear relation for 1-round IDEA as:

$$
\phi \star Z \oplus \psi \star X \oplus \omega \star Y \oplus \lambda=0
$$

where $Z, X$ and $Y$ are round key, input and output of 1-round IDEA, respectively and $\lambda \in \mathbb{Z}_{2}, \phi \star Z=\phi_{1} \cdot \mathbf{Z}_{1} \oplus \ldots \oplus \boldsymbol{\phi}_{6} \cdot \mathbf{Z}_{6}, \psi \star X=\boldsymbol{\psi}_{1} \cdot \mathbf{X}_{1} \oplus \ldots \oplus \boldsymbol{\psi}_{4} \cdot \mathbf{X}_{4}$ and $\omega \star Y=\boldsymbol{\omega}_{1} \cdot \mathbf{Y}_{1} \oplus \ldots \oplus \boldsymbol{\omega}_{4} \cdot \mathbf{Y}_{4}$ such that $\phi=\left(\boldsymbol{\phi}_{1}, \ldots, \boldsymbol{\phi}_{6}\right), \psi=\left(\boldsymbol{\psi}_{1}, \ldots, \boldsymbol{\psi}_{4}\right)$ and $\omega=\left(\boldsymbol{\omega}_{1}, \ldots, \boldsymbol{\omega}_{4}\right)$ for $\boldsymbol{\phi}_{i}, \boldsymbol{\psi}_{i}$ and $\boldsymbol{\omega}_{i} \in \mathbb{Z}_{2}^{16}$. Here $\boldsymbol{\phi}_{i}, \boldsymbol{\psi}_{i}$ and $\boldsymbol{\omega}_{i}$ are masks for $\mathbf{Z}_{i}=v\left(z_{i}\right), \mathbf{X}_{i}=v\left(x_{i}\right)$ and $\mathbf{Y}_{i}=v\left(y_{i}\right)$, respectively and $x_{i}, y_{i}, z_{i} \in \mathbb{Z}_{2^{n}}$.

For the sake of clarity, let us derive the $24^{\text {th }}$ linear relation in Table 1 , one of 15 linear relations found in [2]:

Example 1: Adding first two output of 1-round IDEA, namely $\mathbf{Y}_{1}$ and $\mathbf{Y}_{2}$ (see Fig. 2 in Appendix A), we have

$$
\mathbf{Y}_{1} \oplus \mathbf{Y}_{2}=\left(\mathbf{X}_{1} \oplus \mathbf{Z}_{1}\right) \oplus\left(\mathbf{X}_{3} \boxplus \mathbf{Z}_{3}\right)
$$

When $\mathbf{Z}_{1}=(0, \ldots, 0)$ or $\mathbf{Z}_{1}=(1, \ldots, 1)$, the least significant bit of $\mathbf{Y}_{1}=$ $\mathbf{X}_{1} \odot \mathbf{Z}_{1}$ is $\mathbf{1} \cdot \mathbf{Y}_{1}=\mathbf{1} \cdot \mathbf{X}_{1} \oplus \mathbf{1} \cdot \mathbf{Z}_{1} \oplus 1$ from the Eq. 4 and the least significant bit of $\mathbf{Y}_{3}=\mathbf{X}_{3} \boxplus \mathbf{Z}_{3}$ is $\mathbf{1} \cdot \mathbf{Y}_{3}=\mathbf{1} \cdot \mathbf{X}_{3} \oplus \mathbf{1} \cdot \mathbf{Z}_{3}$ from the Eq. 2. The addition of $\mathbf{1} \cdot \mathbf{Y}_{1}$ and $\mathbf{1} \cdot \mathbf{Y}_{2}$ becomes

$$
\mathbf{1} \cdot \mathbf{Y}_{1} \oplus \mathbf{1} \cdot \mathbf{Y}_{2}=\mathbf{1} \cdot \mathbf{X}_{1} \oplus \mathbf{1} \cdot \mathbf{Z}_{1} \oplus \mathbf{1} \cdot \mathbf{X}_{3} \oplus \mathbf{1} \cdot \mathbf{Z}_{3} \oplus 1
$$

When $\mathbf{Z}_{1}=(0, \ldots, 0)$ or $(1, \ldots, 1)$, one can represent this equation as a linear relation for 1-round IDEA

$$
(\mathbf{1}, \mathbf{0}, \mathbf{1}, \mathbf{0}, \mathbf{0}, \mathbf{0}) \star Z \oplus(\mathbf{1}, \mathbf{0}, \mathbf{1}, \mathbf{0}) \star X \oplus(\mathbf{1}, \mathbf{1}, \mathbf{0}, \mathbf{0}) \star Y \oplus \mathbf{1}=0
$$


Table 1. List of linear relations for 1-round IDEA given in [2] (indicated by ${ }^{*}$ ) and derived. Here $\mathrm{k}$ is a non-negative integer, $-1 \equiv 0 \bmod \left(2^{16}+1\right),-2^{15} \equiv 2^{15}+1 \bmod$ $\left(2^{16}+1\right)$ and $-2 \equiv 2^{16}-1 \bmod \left(2^{16}+1\right)$.

\begin{tabular}{|c|c|c|c|c|c|c|c|c|c|c|c|}
\hline & $\phi$ & $\psi$ & $\omega$ & $\lambda$ & $z_{1}$ & $z_{2}$ & $z_{3}$ & $z_{4}$ & $z_{5}$ & $z_{6}$ & \# of free bits \\
\hline 1 & $*(\mathbf{0}, \mathbf{0}, \mathbf{0}, \mathbf{1}, \mathbf{0}, \mathbf{1})$ & $(\mathbf{0}, \mathbf{0}, \mathbf{0}, \mathbf{1})$ & $(\mathbf{0}, \mathbf{0}, \mathbf{1}, \mathbf{0})$ & 0 & - & - & - & $\mp 1$ & - & $\mp 1$ & 66 \\
\hline 2 & $(\mathbf{0}, \mathbf{0}, \mathbf{0}, \mathbf{1}, \mathbf{0}, \mathbf{1})$ & $(\mathbf{0}, \mathbf{0}, \mathbf{0}, \mathbf{3})$ & $(\mathbf{0}, \mathbf{0}, \mathbf{1}, \mathbf{0})$ & 0 & - & - & - & $\mp 2^{15}$ & - & $\mp 1$ & 66 \\
\hline 3 & $*(\mathbf{0}, \mathbf{0}, \mathbf{1}, \mathbf{0}, \mathbf{1}, \mathbf{1})$ & $(\mathbf{0}, \mathbf{0}, 1, \mathbf{0})$ & $(\mathbf{1}, \mathbf{0}, \mathbf{1}, \mathbf{1})$ & 0 & - & - & - & - & $\mp 1$ & $\mp 1$ & 66 \\
\hline 4 & $(\mathbf{0}, \mathbf{0}, \mathbf{2}, \mathbf{0}, \mathbf{1}, \mathbf{1})$ & $(\mathbf{0}, \mathbf{0}, \mathbf{3}, \mathbf{0})$ & $(\mathbf{3}, \mathbf{0}, \mathbf{1}, \mathbf{1})$ & 1 & $\mp 2$ & - & $2 k$ & - & $\mp 2^{15}$ & $\mp 2$ & 48 \\
\hline 5 & $(\mathbf{0}, \mathbf{0}, \mathbf{2}, \mathbf{1}, \mathbf{1}, \mathbf{1})$ & $(\mathbf{0}, \mathbf{2}, \mathbf{3}, \mathbf{1})$ & $(\mathbf{3}, \mathbf{0}, \mathbf{3}, \mathbf{3})$ & 1 & $\mp 2$ & $2 k$ & $2 k$ & $\mp 2$ & $\mp 2^{15}$ & $\mp 2$ & 31 \\
\hline 6 & $*(\mathbf{0}, \mathbf{0}, \mathbf{1}, \mathbf{1}, \mathbf{1}, \mathbf{0})$ & $(\mathbf{0}, \mathbf{0}, \mathbf{1}, \mathbf{1})$ & $(\mathbf{1}, \mathbf{0}, \mathbf{0}, \mathbf{1})$ & 0 & - & - & - & $\mp 1$ & $\mp 1$ & - & 66 \\
\hline 7 & $(\mathbf{0}, \mathbf{0}, \mathbf{1}, \mathbf{1}, \mathbf{1}, \mathbf{0})$ & $(\mathbf{0}, \mathbf{0}, \mathbf{1}, \mathbf{3})$ & $(\mathbf{1}, \mathbf{0}, \mathbf{0}, \mathbf{1})$ & 0 & - & - & - & $\mp 2^{15}$ & $\mp 1$ & - & 66 \\
\hline 8 & $*(\mathbf{1}, \mathbf{0}, \mathbf{0}, \mathbf{0}, \mathbf{0}, \mathbf{1})$ & $(\mathbf{0}, \mathbf{1}, \mathbf{0}, \mathbf{0})$ & $(\mathbf{0}, \mathbf{0}, \mathbf{0}, \mathbf{1})$ & 1 & - & - & - & - & - & $\mp 1$ & 82 \\
\hline 9 & $*(\mathbf{1}, \mathbf{0}, \mathbf{0}, \mathbf{1}, \mathbf{0}, \mathbf{0})$ & $(\mathbf{0}, \mathbf{1}, \mathbf{0}, \mathbf{1})$ & $(\mathbf{0}, \mathbf{0}, \mathbf{1}, \mathbf{1})$ & 1 & - & - & - & $\mp 1$ & - & - & 81 \\
\hline 10 & $(\mathbf{0}, \mathbf{2}, \mathbf{0}, \mathbf{1}, \mathbf{0}, \mathbf{0})$ & $(\mathbf{0}, \mathbf{3}, \mathbf{0}, \mathbf{1})$ & $(\mathbf{0}, \mathbf{0}, \mathbf{3}, \mathbf{3})$ & 0 & - & $2 k$ & - & $\mp 2$ & - & - & 79 \\
\hline 11 & $(\mathbf{0}, \mathbf{1}, \mathbf{0}, \mathbf{1}, \mathbf{0}, \mathbf{0})$ & $(\mathbf{0}, \mathbf{1}, \mathbf{0}, \mathbf{3})$ & $(\mathbf{0}, \mathbf{0}, \mathbf{3}, \mathbf{3})$ & 1 & - & - & - & $\mp 2^{15}$ & - & - & 81 \\
\hline 12 & $*(\mathbf{0}, \mathbf{1}, \mathbf{1}, \mathbf{0}, \mathbf{1}, \mathbf{0})$ & $(\mathbf{0}, \mathbf{1}, \mathbf{1}, \mathbf{0})$ & $(\mathbf{1}, \mathbf{0}, \mathbf{1}, \mathbf{0})$ & 1 & - & - & - & - & $\mp 1$ & - & 81 \\
\hline 13 & $*(\mathbf{0}, \mathbf{1}, \mathbf{1}, \mathbf{1}, \mathbf{1}, \mathbf{1})$ & $(\mathbf{0}, \mathbf{1}, \mathbf{1}, \mathbf{1})$ & $(\mathbf{1}, \mathbf{0}, \mathbf{0}, \mathbf{0})$ & 1 & - & - & - & $\mp 1$ & $\mp 1$ & $\mp 1$ & 51 \\
\hline 14 & $(\mathbf{0}, \mathbf{1}, \mathbf{1}, \mathbf{1}, \mathbf{1}, \mathbf{1})$ & $(\mathbf{0}, \mathbf{1}, \mathbf{1}, \mathbf{3})$ & $(\mathbf{1}, \mathbf{0}, \mathbf{0}, \mathbf{0})$ & 1 & - & - & - & $\mp 2^{15}$ & $\mp 1$ & $\mp 1$ & 51 \\
\hline 15 & $(\mathbf{0}, \mathbf{1}, \mathbf{2}, \mathbf{1}, \mathbf{1}, \mathbf{1})$ & $(\mathbf{0}, \mathbf{1}, \mathbf{3}, \mathbf{1})$ & $(\mathbf{3}, \mathbf{0}, \mathbf{0}, \mathbf{0})$ & 0 & - & $\mp 2$ & $2 k$ & $\mp 1$ & $\mp 2^{15}$ & $\mp 2$ & 33 \\
\hline 16 & $*(\mathbf{1}, \mathbf{0}, \mathbf{0}, \mathbf{0}, \mathbf{0}, \mathbf{1})$ & $(\mathbf{1}, \mathbf{0}, \mathbf{0}, \mathbf{0})$ & $(\mathbf{0}, \mathbf{1}, \mathbf{1}, \mathbf{1})$ & 1 & $\mp 1$ & - & - & - & $\mp 1$ & $\mp 1$ & 51 \\
\hline 17 & $(\mathbf{1}, \mathbf{0}, \mathbf{0}, \mathbf{0}, \mathbf{0}, \mathbf{1})$ & $(\mathbf{1}, \mathbf{0}, \mathbf{0}, \mathbf{0})$ & $(\mathbf{0}, \mathbf{3}, \mathbf{1}, \mathbf{1})$ & 1 & $\mp 2$ & - & $2 k$ & - & $\mp 2^{15}$ & $\mp 1$ & 49 \\
\hline 18 & $*(\mathbf{1}, \mathbf{0}, \mathbf{0}, \mathbf{1}, \mathbf{1}, \mathbf{0})$ & $(\mathbf{1}, \mathbf{0}, \mathbf{0}, \mathbf{1})$ & $(\mathbf{0}, \mathbf{1}, \mathbf{0}, \mathbf{1})$ & 1 & $\mp 1$ & - & - & $\mp 1$ & $\mp 1$ & - & 51 \\
\hline 19 & $(\mathbf{1}, \mathbf{0}, \mathbf{0}, \mathbf{1}, \mathbf{1}, \mathbf{0})$ & $(\mathbf{1}, \mathbf{0}, \mathbf{0}, \mathbf{3})$ & $(\mathbf{0}, \mathbf{1}, \mathbf{0}, \mathbf{1})$ & 1 & $\mp 1$ & - & - & $\mp 2^{15}$ & $\mp 1$ & - & 51 \\
\hline 20 & $(\mathbf{1}, \mathbf{0}, \mathbf{0}, \mathbf{1}, \mathbf{1}, \mathbf{0})$ & $(\mathbf{3}, \mathbf{0}, \mathbf{0}, \mathbf{1})$ & $(\mathbf{0}, \mathbf{1}, \mathbf{0}, \mathbf{1})$ & 1 & $\mp 2^{15}$ & - & - & $\mp 1$ & $\mp 1$ & - & 51 \\
\hline 21 & $(\mathbf{1}, \mathbf{0}, \mathbf{0}, \mathbf{1}, \mathbf{1}, \mathbf{0})$ & $(3, \mathbf{0}, \mathbf{0}, \mathbf{3})$ & $(\mathbf{0}, \mathbf{1}, \mathbf{0}, \mathbf{1})$ & 1 & $\mp 2^{15}$ & - & - & $\mp 2^{15}$ & $\mp 1$ & - & 51 \\
\hline 22 & $(\mathbf{1}, \mathbf{0}, \mathbf{2}, \mathbf{1}, \mathbf{1}, \mathbf{0})$ & $(\mathbf{1}, \mathbf{0}, \mathbf{2}, \mathbf{1})$ & $(\mathbf{0}, \mathbf{1}, \mathbf{0}, \mathbf{1})$ & 0 & $\mp 2$ & - & $2 k$ & $\mp 1$ & $\mp 2^{15}$ & - & 49 \\
\hline 23 & $(\mathbf{1}, \mathbf{0}, \mathbf{2}, \mathbf{1}, \mathbf{1}, \mathbf{0})$ & $(\mathbf{1}, \mathbf{0}, \mathbf{2}, \mathbf{3})$ & $(\mathbf{0}, \mathbf{1}, \mathbf{0}, \mathbf{1})$ & 0 & $\mp 2$ & - & $2 k$ & $\mp 2^{15}$ & $\mp 2^{15}$ & - & 49 \\
\hline 24 & $*(\mathbf{1}, \mathbf{0}, \mathbf{1}, \mathbf{0}, \mathbf{0}, \mathbf{0})$ & $(\mathbf{1}, \mathbf{0}, \mathbf{1}, \mathbf{0})$ & $(\mathbf{1}, \mathbf{1}, \mathbf{0}, \mathbf{0})$ & 1 & $\mp 1$ & - & - & - & - & - & 81 \\
\hline 25 & $(\mathbf{1}, \mathbf{0}, \mathbf{2}, \mathbf{0}, \mathbf{0}, \mathbf{0})$ & $(\mathbf{1}, \mathbf{0}, \mathbf{3}, \mathbf{0})$ & $(\mathbf{3}, \mathbf{3}, \mathbf{0}, \mathbf{0})$ & 0 & $\mp 2$ & - & $2 k$ & - & - & - & 79 \\
\hline 26 & $(\mathbf{1}, \mathbf{0}, \mathbf{1}, \mathbf{0}, \mathbf{0}, \mathbf{0})$ & $(\mathbf{3}, \mathbf{0}, \mathbf{1}, \mathbf{0})$ & $(\mathbf{1}, \mathbf{1}, \mathbf{0}, \mathbf{0})$ & 1 & $\mp 2^{15}$ & - & - & - & - & - & 81 \\
\hline 27 & $*(\mathbf{1}, \mathbf{0}, \mathbf{1}, \mathbf{1}, \mathbf{0}, \mathbf{1})$ & $(\mathbf{1}, \mathbf{0}, \mathbf{1}, \mathbf{1})$ & $(\mathbf{1}, \mathbf{1}, \mathbf{1}, \mathbf{0})$ & 1 & $\mp 1$ & - & - & $\mp 1$ & - & $\mp 1$ & 51 \\
\hline 28 & $(\mathbf{1}, \mathbf{0}, \mathbf{1}, \mathbf{1}, \mathbf{0}, \mathbf{1})$ & $(\mathbf{1}, \mathbf{0}, \mathbf{1}, \mathbf{3})$ & $(\mathbf{1}, \mathbf{1}, \mathbf{1}, \mathbf{0})$ & 1 & $\mp 1$ & - & - & $\mp 2^{15}$ & - & $\mp 1$ & 51 \\
\hline 29 & $(\mathbf{1}, \mathbf{0}, \mathbf{2}, \mathbf{1}, \mathbf{0}, \mathbf{1})$ & $(\mathbf{1}, \mathbf{0}, \mathbf{3}, \mathbf{1})$ & $(\mathbf{3}, \mathbf{3}, \mathbf{3}, \mathbf{0})$ & 0 & $\mp 2$ & - & $2 k$ & $\mp 1$ & - & $\mp 1$ & 49 \\
\hline 30 & $(\mathbf{1}, \mathbf{0}, \mathbf{2}, \mathbf{1}, \mathbf{0}, \mathbf{1})$ & $(\mathbf{1}, \mathbf{0}, \mathbf{3}, \mathbf{3})$ & $(\mathbf{3}, \mathbf{3}, \mathbf{3}, \mathbf{0})$ & 0 & $\mp 2$ & - & $2 k$ & $\mp 2^{15}$ & - & $\mp 1$ & 49 \\
\hline 31 & $(\mathbf{1}, \mathbf{0}, \mathbf{1}, \mathbf{1}, \mathbf{0}, \mathbf{1})$ & $(\mathbf{3}, \mathbf{0}, \mathbf{1}, \mathbf{1})$ & $(\mathbf{1}, \mathbf{1}, \mathbf{1}, \mathbf{0})$ & 1 & $\mp 2^{15}$ & - & - & $\mp 1$ & - & $\mp 1$ & 51 \\
\hline 32 & $(\mathbf{1}, \mathbf{0}, \mathbf{1}, \mathbf{1}, \mathbf{0}, \mathbf{1})$ & $(\mathbf{3}, \mathbf{0}, \mathbf{1}, \mathbf{3})$ & $(\mathbf{1}, \mathbf{1}, \mathbf{1}, \mathbf{0})$ & 1 & $\mp 2^{15}$ & - & - & $\mp 2^{15}$ & - & $\mp 1$ & 51 \\
\hline 33 & $*(\mathbf{1}, \mathbf{1}, \mathbf{0}, \mathbf{0}, \mathbf{1}, \mathbf{0})$ & $(\mathbf{1}, \mathbf{1}, \mathbf{0}, \mathbf{0})$ & $(\mathbf{0}, \mathbf{1}, \mathbf{1}, \mathbf{0})$ & 0 & $\mp 1$ & - & - & - & $\mp 1$ & - & 66 \\
\hline 34 & $(\mathbf{1}, \mathbf{1}, \mathbf{0}, \mathbf{0}, \mathbf{1}, \mathbf{0})$ & $(\mathbf{3}, \mathbf{1}, \mathbf{0}, \mathbf{0})$ & $(\mathbf{0}, \mathbf{1}, \mathbf{1}, \mathbf{0})$ & 0 & $\mp 2^{15}$ & - & - & - & $\mp 1$ & - & 66 \\
\hline 35 & $(\mathbf{1}, \mathbf{1}, \mathbf{2}, \mathbf{0}, \mathbf{1}, \mathbf{0})$ & $(\mathbf{1}, \mathbf{1}, \mathbf{2}, \mathbf{0})$ & $(\mathbf{0}, \mathbf{1}, \mathbf{1}, \mathbf{0})$ & 1 & $\mp 2$ & - & $2 k$ & - & $\mp 2^{15}$ & - & 64 \\
\hline 36 & $*(\mathbf{1}, \mathbf{1}, \mathbf{0}, \mathbf{1}, \mathbf{1}, \mathbf{1})$ & $(\mathbf{1}, \mathbf{1}, \mathbf{0}, \mathbf{1})$ & $(\mathbf{0}, \mathbf{1}, \mathbf{0}, \mathbf{0})$ & 0 & $\mp 1$ & - & - & $\mp 1$ & $\mp 1$ & $\mp 1$ & 36 \\
\hline 37 & $(\mathbf{1}, \mathbf{1}, \mathbf{2}, \mathbf{1}, \mathbf{1}, \mathbf{1})$ & $(\mathbf{1}, \mathbf{1}, \mathbf{2}, \mathbf{1})$ & $(\mathbf{0}, \mathbf{1}, \mathbf{0}, \mathbf{0})$ & 1 & $\mp 2$ & - & $2 k$ & $\mp 1$ & $\mp 2^{15}$ & $\mp 1$ & 34 \\
\hline 38 & $(\mathbf{1}, \mathbf{1}, \mathbf{2}, \mathbf{1}, \mathbf{1}, \mathbf{1})$ & $(\mathbf{1}, \mathbf{1}, \mathbf{2}, \mathbf{3})$ & $(\mathbf{0}, \mathbf{1}, \mathbf{0}, \mathbf{0})$ & 1 & $\mp 2$ & - & $2 k$ & $\mp 2^{15}$ & $\mp 2^{15}$ & $\mp 1$ & 34 \\
\hline 39 & $(\mathbf{1}, \mathbf{1}, \mathbf{0}, \mathbf{1}, \mathbf{1}, \mathbf{1})$ & $(\mathbf{3}, \mathbf{1}, \mathbf{0}, \mathbf{1})$ & $(\mathbf{0}, \mathbf{1}, \mathbf{0}, \mathbf{0})$ & 0 & $\mp 2^{15}$ & - & - & $\mp 1$ & $\mp 1$ & $\mp 1$ & 36 \\
\hline
\end{tabular}


Table 1. (continued)

\begin{tabular}{l|l|l|l|l|l|l|l|l|l|l|l}
\hline & $\phi$ & $\psi$ & $\omega$ & $\lambda$ & $z_{1}$ & $z_{2}$ & $z_{3}$ & $z_{4}$ & $z_{5}$ & $z_{6}$ & \# of free bits \\
\hline 40 & $(\mathbf{1}, \mathbf{1}, \mathbf{0}, \mathbf{1}, \mathbf{1}, \mathbf{1})$ & $(\mathbf{3}, \mathbf{1}, \mathbf{0}, \mathbf{3})$ & $(\mathbf{0}, \mathbf{1}, \mathbf{0}, \mathbf{0})$ & 0 & $\mp 2^{15}$ & - & - & $\mp 2^{15}$ & $\mp 1$ & $\mp 1$ & 36 \\
\hline 41 & $(\mathbf{1}, \mathbf{1}, \mathbf{0}, \mathbf{1}, \mathbf{1}, \mathbf{1})$ & $(\mathbf{1}, \mathbf{1}, \mathbf{0}, \mathbf{1})$ & $(\mathbf{0}, \mathbf{3}, \mathbf{0}, \mathbf{0})$ & 0 & $\mp 2$ & - & - & $\mp 1$ & $\mp 2^{15}$ & $\mp 2$ & 34 \\
\hline 42 & $(\mathbf{1}, \mathbf{1}, \mathbf{0}, \mathbf{1}, \mathbf{1}, \mathbf{1})$ & $(\mathbf{1}, \mathbf{1}, \mathbf{0}, \mathbf{3})$ & $(\mathbf{0}, \mathbf{3}, \mathbf{0}, \mathbf{0})$ & 0 & $\mp 2$ & - & - & $\mp 2^{15}$ & $\mp 2^{15}$ & $\mp 2$ & 34 \\
\hline 43 & $*(\mathbf{1}, \mathbf{1}, \mathbf{1}, \mathbf{0}, \mathbf{0}, \mathbf{1})$ & $(\mathbf{1}, \mathbf{1}, \mathbf{1}, \mathbf{0})$ & $(\mathbf{1}, \mathbf{1}, \mathbf{0}, \mathbf{1})$ & 0 & $\mp 1$ & - & - & - & - & $\mp 1$ & 66 \\
\hline 44 & $(\mathbf{1}, \mathbf{1}, \mathbf{1}, \mathbf{0}, \mathbf{0}, \mathbf{1})$ & $(\mathbf{3}, \mathbf{1}, \mathbf{1}, \mathbf{0})$ & $(\mathbf{1}, \mathbf{1}, \mathbf{0}, \mathbf{1})$ & 0 & $\mp 2^{15}$ & - & - & - & - & $\mp 1$ & 66 \\
\hline 45 & $(\mathbf{1}, \mathbf{1}, \mathbf{2}, \mathbf{0}, \mathbf{0}, \mathbf{1})$ & $(\mathbf{1}, \mathbf{1}, \mathbf{3}, \mathbf{0})$ & $(\mathbf{3}, \mathbf{3}, \mathbf{0}, \mathbf{1})$ & 1 & $\mp 2$ & - & $2 k$ & - & - & $\mp 1$ & 64 \\
\hline 46 & $*(\mathbf{1}, \mathbf{1}, \mathbf{1}, \mathbf{1}, \mathbf{0})$ & $(\mathbf{1}, \mathbf{1}, \mathbf{1}, \mathbf{1})$ & $(\mathbf{1}, \mathbf{1}, \mathbf{1}, \mathbf{1})$ & 0 & $\mp 1$ & - & - & $\mp 1$ & - & - & 66 \\
\hline 47 & $(\mathbf{1}, \mathbf{1}, \mathbf{1}, \mathbf{1}, \mathbf{0})$ & $(\mathbf{1}, \mathbf{1}, \mathbf{1}, \mathbf{3})$ & $(\mathbf{1}, \mathbf{1}, \mathbf{1}, \mathbf{1})$ & 0 & $\mp 1$ & - & - & $\mp 2^{15}$ & - & - & 66 \\
\hline 48 & $(\mathbf{1}, \mathbf{1}, \mathbf{1}, \mathbf{1}, \mathbf{0}, \mathbf{0})$ & $(\mathbf{3}, \mathbf{1}, \mathbf{1}, \mathbf{1})$ & $(\mathbf{1}, \mathbf{1}, \mathbf{1}, \mathbf{1})$ & 0 & $\mp 2^{15}$ & - & - & $\mp 1$ & - & - & 66 \\
\hline 49 & $(\mathbf{1}, \mathbf{1}, \mathbf{1}, \mathbf{1}, \mathbf{0}, \mathbf{0})$ & $(\mathbf{3}, \mathbf{1}, \mathbf{1}, \mathbf{3})$ & $(\mathbf{1}, \mathbf{1}, \mathbf{1}, \mathbf{1})$ & 0 & $\mp 2^{15}$ & - & - & $\mp 2^{15}$ & - & - & 66 \\
\hline 50 & $(\mathbf{1}, \mathbf{1}, \mathbf{2}, \mathbf{1}, \mathbf{0}, \mathbf{0})$ & $(\mathbf{1}, \mathbf{1}, \mathbf{3}, \mathbf{1})$ & $(\mathbf{3}, \mathbf{3}, \mathbf{1}, \mathbf{1})$ & 1 & $\mp 2$ & - & $2 k$ & $\mp 1$ & - & - & 64 \\
\hline 51 & $(\mathbf{1}, \mathbf{1}, \mathbf{2}, \mathbf{1}, \mathbf{0}, \mathbf{0})$ & $(\mathbf{1}, \mathbf{1}, \mathbf{3}, \mathbf{3})$ & $(\mathbf{3}, \mathbf{3}, \mathbf{1}, \mathbf{1})$ & 1 & $\mp 2$ & - & $2 k$ & $\mp 2^{15}$ & - & - & 64 \\
\hline 52 & $(\mathbf{1}, \mathbf{2}, \mathbf{1}, \mathbf{1}, \mathbf{0}, \mathbf{0})$ & $(\mathbf{1}, \mathbf{3}, \mathbf{1}, \mathbf{1})$ & $(\mathbf{1}, \mathbf{1}, \mathbf{3}, \mathbf{3})$ & 1 & $\mp 1$ & $2 k$ & - & $\mp 2$ & - & - & 64 \\
\hline 53 & $(\mathbf{1}, \mathbf{2}, \mathbf{1}, \mathbf{1}, \mathbf{0}, \mathbf{0})$ & $(\mathbf{3}, \mathbf{3}, \mathbf{1}, \mathbf{1})$ & $(\mathbf{1}, \mathbf{1}, \mathbf{3}, \mathbf{3})$ & 1 & $\mp 2^{15}$ & $2 k$ & - & $\mp 2$ & - & - & 64 \\
\hline 54 & $(\mathbf{1}, \mathbf{2}, \mathbf{2}, \mathbf{1}, \mathbf{0}, \mathbf{0})$ & $(\mathbf{1}, \mathbf{3}, \mathbf{3}, \mathbf{1})$ & $(\mathbf{3}, \mathbf{3}, \mathbf{3}, \mathbf{3})$ & 1 & $\mp 2$ & $2 k$ & $2 k$ & $\mp 2$ & - & - & 62 \\
\hline
\end{tabular}

Example 2: From the Table 1, when $\mathbf{Z}_{j}=v\left(z_{j}\right), z_{1}=\mp 2, z_{4}=\mp 2^{15}, z_{5}=$ $\mp 2^{15}$ and $z_{6}=\mp 2$ for $\phi=(\mathbf{1}, \mathbf{1}, \mathbf{0}, \mathbf{1}, \mathbf{1}, \mathbf{1}), \psi=(\mathbf{1}, \mathbf{1}, \mathbf{0}, \mathbf{3}), \omega=(\mathbf{0}, \mathbf{3}, \mathbf{0}, \mathbf{0})$ and $\lambda=0$ we have

$\mathbf{1} \cdot \mathbf{Z}_{1} \oplus \mathbf{1} \cdot \mathbf{Z}_{2} \oplus \mathbf{1} \cdot \mathbf{Z}_{4} \oplus \mathbf{1} \cdot \mathbf{Z}_{5} \oplus \mathbf{1} \cdot \mathbf{Z}_{6} \oplus \mathbf{1} \cdot \mathbf{X}_{1} \oplus \mathbf{1} \cdot \mathbf{X}_{2} \oplus \mathbf{3} \cdot \mathbf{X}_{4}=\mathbf{3} \cdot \mathbf{Y}_{2}$

This relation, one of new 39 linear relations derived, is the $42^{t h}$ linear relation in Table 1.

\subsection{New Linear Relations Algorithmically Generated}

Let us consider the $35^{\text {th }}$ and the $45^{\text {th }}$ linear relations for 1-round IDEA in Table 1 to obtain a new relation which is not listed in Table 1.

For the $35^{\text {th }}$ linear relation $(\mathbf{1}, \mathbf{1}, \mathbf{2}, \mathbf{0}) \rightarrow(\mathbf{0}, \mathbf{1}, \mathbf{1}, \mathbf{0})$ with key subblocks restrictions $z_{1}=\mp 2, z_{3}=2 k$ and $z_{5}=\mp 2^{15}$ and the $45^{\text {th }}$ linear relation $(\mathbf{1}, \mathbf{1}, \mathbf{3}, \mathbf{0}) \rightarrow(\mathbf{3}, \mathbf{3}, \mathbf{0}, \mathbf{1})$ with restrictions $z_{1}=\mp 2, z_{3}=2 k$ and $z_{6}=\mp 1$, we have two corresponding Eqs. (8) and (9) respectively

$\mathbf{1} \cdot \mathbf{Z}_{1} \oplus \mathbf{1} \cdot \mathbf{Z}_{2} \oplus \mathbf{2} \cdot \mathbf{Z}_{3} \oplus \mathbf{1} \cdot \mathbf{Z}_{5} \oplus \mathbf{1} \cdot \mathbf{X}_{1} \oplus \mathbf{1} \cdot \mathbf{X}_{2} \oplus \mathbf{2} \cdot \mathbf{X}_{3} \oplus \mathbf{1} \cdot \mathbf{Y}_{2} \oplus \mathbf{1} \cdot \mathbf{Y}_{3} \oplus \mathbf{1}=0$

$\mathbf{1} \cdot \mathbf{Z}_{1} \oplus \mathbf{1} \cdot \mathbf{Z}_{2} \oplus \mathbf{2} \cdot \mathbf{Z}_{3} \oplus \mathbf{1} \cdot \mathbf{Z}_{6} \oplus \mathbf{1} \cdot \mathbf{X}_{1} \oplus \mathbf{1} \cdot \mathbf{X}_{2} \oplus \mathbf{3} \cdot \mathbf{X}_{3} \oplus \mathbf{3} \cdot \mathbf{Y}_{1} \oplus \mathbf{3} \cdot \mathbf{Y}_{2} \oplus \mathbf{1} \cdot \mathbf{Y}_{4} \oplus \mathbf{1}=0$

Equations (8) and (9) key subblocks restrictions do not give any conflicts and they can be combined (by adding them in mod 2) to obtain the following linear relation candidate:

$$
\mathbf{1} \cdot \mathbf{Z}_{5} \oplus \mathbf{1} \cdot \mathbf{Z}_{6} \oplus \mathbf{1} \cdot \mathbf{X}_{3} \oplus \mathbf{3} \cdot \mathbf{Y}_{1} \oplus \mathbf{2} \cdot \mathbf{Y}_{2} \oplus \mathbf{1} \cdot \mathbf{Y}_{3} \oplus \mathbf{1} \cdot \mathbf{Y}_{4} \oplus \mathbf{1}=0
$$


We have used many inputs for 1-round IDEA to check that linear relation in (10) holds with probability one under the key subblocks restrictions $z_{1}=\mp 2$, $z_{3}=2 k, z_{5}=\mp 2^{15}$ and $z_{6}=\mp 1$. In fact, we have observed that only key restrictions $z_{5}=\mp 2^{15}$ and $z_{6}=\mp 1$ are enough to make this linear relation hold with probability one according to our experiments. Hence we have devised a new algorithm to find new linear relations for 1-round IDEA based on a set of 54 linear relations for 1-round IDEA in Table 1. Considering these known linear relations, we found additional 201 new linear relations for 1-round IDEA (see Table 5, Appendix B) using the following algorithm:

Algorithm 1. An algorithm for finding new linear relations for 1-round IDEA based on existing linear ones:

Let $\mathcal{S}$ be the set of linear relations with their key subblocks restrictions.

Step 1 All pair of $\mathcal{S}$ whose key subblocks values coincided are chosen.

Step 2 Any chosen pairs are also combined (directly added in mod 2).

Step 3 Each linear relation candidates in Step 2 is tested using 10 million test vectors to check whether it is a linear relation or not.

Step 4 The ones (i.e. candidate linear relations) passing Step 3 added to $\mathcal{S}$.

Step 5 Previous steps are repeated until there is no increase in the number of the elements of the set $\mathcal{S}$.

Step 6 Key restrictions of each linear relation in $\mathcal{S}$ are checked to remove unnecessary restrictions using 50000 test vectors.

We note that the last step has been added as a result of comments provided by Nakahara [7]. All 54 linear relations in Table 1 can be derived by hand calculation considering all combinations of subblock outputs of 1-round IDEA, $\mathbf{Y}_{i}$ and subblock keys of 1-round IDEA, $\mathbf{Z}_{i}$ which give us linear relations for the operations used in IDEA cipher. By using Algorithm 1, it is possible to obtain linear relations that can not be derived in this way.

\section{Linear Weak Key Classes for IDEA}

As indicated in Table 2, three linear relations, namely the $24^{t h}$, the $33^{\text {th }}$ and the $12^{\text {th }}$ relations in Table 1 were successively used to find a linear relation for 8,5-round IDEA holding with probability one [2]. Because of key subblocks restrictions done in each round, this linear relation is satisfied for all 64-bit plaintexts provided that ranges of zero key bits' indices of a 128-bit master key bits are between 0-25, 29-71, and 75-110. Such key is a member of a class of weak keys with size $2^{23}$ since each of the remaining 23 bits of the master key can take 0 or 1 .

Note that this has been the largest known class of weak keys based on a linear relation for 8,5-round IDEA. Hence this linear relation can be regarded as the best linear relation for 8,5-round IDEA. Based on this linear relation, we have found a new class of weak keys with cardinality $2^{24}$. For this construction, we replace the first round linear relation $(\mathbf{1}, \mathbf{0}, \mathbf{1}, \mathbf{0}) \rightarrow(\mathbf{1}, \mathbf{1}, \mathbf{0}, \mathbf{0})$ with $(\{\mathbf{1}, \mathbf{3}\}, \mathbf{0}, \mathbf{1}, \mathbf{0}) \rightarrow(\mathbf{1}, \mathbf{1}, \mathbf{0}, \mathbf{0})$ (see Table 3$)$. For the former and latter relations, $\mathbf{Z}_{1}^{(1)}$ is chosen $\mathbf{0}=(0, \ldots, 0)$ or $\mathbf{1}=(1, \ldots, 1)$ and $\mathbf{Z}_{1}^{(1)}$ is restricted 
Table 2. Each round linear relation and ranges for indices of zero key bits of IDEA master key are considered to derive the linear relation $(\mathbf{1}, \mathbf{0}, \mathbf{1}, \mathbf{0}) \rightarrow(\mathbf{0}, \mathbf{1}, \mathbf{1}, \mathbf{0})$ for 8,5 -round IDEA satisfied by a linear weak key class with cardinality $2^{23}$.

\begin{tabular}{l|l|l|l}
\hline Round $i$ & Linear relation $\psi \rightarrow \omega$ & $\mathbf{Z}_{1}^{(i)}$ & $\mathbf{Z}_{5}^{(i)}$ \\
\hline 1 & $(\mathbf{1}, \mathbf{0}, \mathbf{1}, \mathbf{0}) \rightarrow(\mathbf{1}, \mathbf{1}, \mathbf{0}, \mathbf{0})$ & $0-14$ & - \\
\hline 2 & $(\mathbf{1}, \mathbf{1}, \mathbf{0}, \mathbf{0}) \rightarrow(\mathbf{0}, \mathbf{1}, \mathbf{1}, \mathbf{0})$ & $96-110$ & $57-71$ \\
\hline 3 & $(\mathbf{0}, \mathbf{1}, \mathbf{1}, \mathbf{0}) \rightarrow(\mathbf{1}, \mathbf{0}, \mathbf{1}, \mathbf{0})$ & - & $50-64$ \\
\hline 4 & $(\mathbf{1}, \mathbf{0}, \mathbf{1}, \mathbf{0}) \rightarrow(\mathbf{1}, \mathbf{1}, \mathbf{0}, \mathbf{0})$ & $82-96$ & - \\
\hline 5 & $(\mathbf{1}, \mathbf{1}, \mathbf{0}, \mathbf{0}) \rightarrow(\mathbf{0}, \mathbf{1}, \mathbf{1}, \mathbf{0})$ & $75-89$ & $11-25$ \\
\hline 6 & $(\mathbf{0}, \mathbf{1}, \mathbf{1}, \mathbf{0}) \rightarrow(\mathbf{1}, \mathbf{0}, \mathbf{1}, \mathbf{0})$ & - & $4-18$ \\
\hline 7 & $(\mathbf{1}, \mathbf{0}, \mathbf{1}, \mathbf{0}) \rightarrow(\mathbf{1}, \mathbf{1}, \mathbf{0}, \mathbf{0})$ & $36-50$ & - \\
\hline 8 & $(\mathbf{1}, \mathbf{1}, \mathbf{0}, \mathbf{0}) \rightarrow(\mathbf{0}, \mathbf{1}, \mathbf{1}, \mathbf{0})$ & $29-44$ & $93-107$ \\
\hline 8,5 & $(\mathbf{0}, \mathbf{1}, \mathbf{1}, \mathbf{0}) \rightarrow(\mathbf{0}, \mathbf{1}, \mathbf{1}, \mathbf{0})$ & - & - \\
\hline
\end{tabular}

Table 3. Each round linear relation and ranges for indices of zero key bits of IDEA master key are considered to derive the linear relation $(\{\mathbf{1}, \mathbf{3}\}, \mathbf{0}, \mathbf{1}, \mathbf{0}) \rightarrow(\mathbf{0}, \mathbf{1}, \mathbf{1}, \mathbf{0})$ for 8,5 -round IDEA satisfied by a linear weak key class with cardinality $2^{24}$.

\begin{tabular}{l|l|l|l}
\hline Round $i$ & Linear relation $\psi \rightarrow \omega$ & $\mathbf{Z}_{1}^{(i)}$ & $\mathbf{Z}_{5}^{(i)}$ \\
\hline 1 & $(\{\mathbf{1}, \mathbf{3}\}, \mathbf{0}, \mathbf{1}, \mathbf{0}) \rightarrow(\mathbf{1}, \mathbf{1}, \mathbf{0}, \mathbf{0})$ & $1-15$ & - \\
\hline 2 & $(\mathbf{1}, \mathbf{1}, \mathbf{0}, \mathbf{0}) \rightarrow(\mathbf{0}, \mathbf{1}, \mathbf{1}, \mathbf{0})$ & $96-110$ & $57-71$ \\
\hline 3 & $(\mathbf{0}, \mathbf{1}, \mathbf{1}, \mathbf{0}) \rightarrow(\mathbf{1}, \mathbf{0}, \mathbf{1}, \mathbf{0})$ & - & $50-64$ \\
\hline 4 & $(\mathbf{1}, \mathbf{0}, \mathbf{1}, \mathbf{0}) \rightarrow(\mathbf{1}, \mathbf{1}, \mathbf{0}, \mathbf{0})$ & $82-96$ & - \\
\hline 5 & $(\mathbf{1}, \mathbf{1}, \mathbf{0}, \mathbf{0}) \rightarrow(\mathbf{0}, \mathbf{1}, \mathbf{1}, \mathbf{0})$ & $75-89$ & $11-25$ \\
\hline 6 & $(\mathbf{0}, \mathbf{1}, \mathbf{1}, \mathbf{0}) \rightarrow(\mathbf{1}, \mathbf{0}, \mathbf{1}, \mathbf{0})$ & - & $4-18$ \\
\hline 7 & $(\mathbf{1}, \mathbf{0}, \mathbf{1}, \mathbf{0}) \rightarrow(\mathbf{1}, \mathbf{1}, \mathbf{0}, \mathbf{0})$ & $36-50$ & - \\
\hline 8 & $(\mathbf{1}, \mathbf{1}, \mathbf{0}, \mathbf{0}) \rightarrow(\mathbf{0}, \mathbf{1}, \mathbf{1}, \mathbf{0})$ & $29-44$ & $93-107$ \\
\hline 8,5 & $(\mathbf{0}, \mathbf{1}, \mathbf{1}, \mathbf{0}) \rightarrow(\mathbf{0}, \mathbf{1}, \mathbf{1}, \mathbf{0})$ & - & - \\
\hline
\end{tabular}

to $\mathbf{0}$ or $\mathbf{2}^{15}$, respectively. Note that $(\{\mathbf{1}, \mathbf{3}\}, \mathbf{0}, \mathbf{1}, \mathbf{0})=(\mathbf{1}, \mathbf{0}, \mathbf{1}, \mathbf{0})$ (respectively $(\{\mathbf{1}, \mathbf{3}\}, \mathbf{0}, \mathbf{1}, \mathbf{0})=(\mathbf{3}, \mathbf{0}, \mathbf{1}, \mathbf{0}))$ if $\mathbf{Z}_{1}^{(1)}$ is equal to $\mathbf{0}$ (respectively $\mathbf{Z}_{1}^{(1)}=\mathbf{2}^{\mathbf{1 5}}$ ). Therefore, zero key bits' indices of a 128-bit key are between 1-25, 29-71, and 75110. Then linear relation $(\{\mathbf{1}, \mathbf{3}\}, \mathbf{0}, \mathbf{1}, \mathbf{0}) \rightarrow(\mathbf{0}, \mathbf{1}, \mathbf{1}, \mathbf{0})$ for the 8,5 -round IDEA holds with probability one (Table 3 ) and there are $2^{24}$ such keys. We haven't discovered other linear relations in Tables 1 and 5 similar to the best linear relation giving a large class of weak keys because of the following reasons:

- If we compare Table 1 with Table 5 in Appendix B, then it can be seen that for most cases, linear relations in Table 1 derived in [2] have less key restrictions than others.

- In Table 1, each of linear relations numbered with 8, 9, 12, 24, 26 has one key subblock restriction and each of linear relations numbered with $1,2,3,6,7,10$, 
$25,34,43,44,46,47,48,49$ has two key subblocks restrictions. There aren't any linear relations with one key subblock restriction in Table 5, but there are linear relations numbered with $98,125,159,185$ and 216 having two key subblocks restrictions in Table 5 . In order to find a linear relation for 8,5-round IDEA providing a large class of weak keys, it is better to use those relations (with less key subblocks restrictions) listed above. However, it is not possible to derive such linear relation for 8,5-round IDEA using these relations and linear relations with key subblocks $\mp 2$ or $\mp 2^{15}$ restrictions other than those derived in [2] in both Tables 1 and 5. Because

(i) we faced with key subblocks restrictions giving conflicts, that is, some bits of the master 128-bit of IDEA are both 0 and 1 due to key subblocks restrictions of two linear relations considered for two different rounds, especially when a key subblock of one linear relation is equal to 0 or 1 and a key subblock of other one is chosen as $\mp 2$ or $\mp 2^{15}$;

(ii) we haven't found successive linear relations for many linear relations with key subblock restriction like $\mp 2$ or $\mp 2^{15}$ while deriving multi round linear relation. For example, for the $75^{\text {th }}$ linear relation in Table 5, namely $(\mathbf{3}, \mathbf{3}, \mathbf{0}, \mathbf{1}) \rightarrow(\mathbf{2}, \mathbf{3}, \mathbf{2}, \mathbf{2})$ there aren't any linear relations whose input mask is equal to $(\mathbf{2}, \mathbf{3}, \mathbf{2}, \mathbf{2})$ in both Tables 1 and 5 .

Because these limitations to derive new linear relations the block cipher, we assume that key subblocks (subkeys) are independent. Then under weakkey assumptions we consider each linear relation for 1-round IDEA cipher from Tables 1 and 5 as two vertices connected by a single edge having a direction. In this manner we have a directed graph and using suitable functions of Digraph module from SageMath [11] we find many paths with length 8 and then consider last 0.5 round's relations in order to get 438 linear relations for 8.5round IDEA cipher. In Table 6 (Appendix B), 50 of them with less number of key bits restriction for the master key, whose size is 832-bits (considering all 52 16-bit key subblocks) are listed. Note that second relation in this table $(\mathbf{1}, \mathbf{1}, \mathbf{0}, \mathbf{0}) \longrightarrow(\mathbf{3}, \mathbf{1}, \mathbf{0}, \mathbf{0})$ is a linear relation for 8.5 -round IDEA cipher and associated with a class of weak keys with the cardinality $2^{586}$ whenever key subblocks (subkeys) are chosen independently. Note that the key space with size $2^{832}$ is extremely larger than this class.

\section{Conclusion}

In this paper we give several new properties on the nonlinearity of the multiplication operation $\odot$. Using its invariance properties, it is possible to calculate the nonlinearity just for one value of the associated vector function to learn one, two or three different values giving the same the nonlinearity. Furthermore, we give an upper bound for its nonlinearity when values are power of two. It is low for small powers. In fact, it is expected that the nonlinearity of such building blocks of block ciphers should be high. We devise an algorithm to find a new set of linear relations for 1-round IDEA using a set of linear relations directly derived and a set of known linear relations. We present one linear weak key class 
slightly bigger than one known in the literature. Assuming that all key subblocks are chosen independently, we generate a new set of linear relations for full IDEA cipher using linear relations for 1-round IDEA. All these findings extend the related work done by Daemen et al. and they are meaningful to understand how properties of building components of a cipher are related to its security.

\section{A Appendix: IDEA Block Cipher}

The graph of the encryption of IDEA can be seen in Fig. 1. The key scheduling algorithm and the list of all 16-bit key subblocks (Table 4) are given in Appendix.

\section{A.1 Key Schedule and Decryption Algorithm}

For a given 128-bit key, 52 16-bit key subblocks are generated for the encryption. For the construction of these subblocks, the first step is to partition given 128-bit key into 8 pieces and assign them as the first 8 key subblocks of the 52 subblocks: $\mathbf{Z}_{1}^{(1)}, \mathbf{Z}_{2}^{(1)}, . ., \mathbf{Z}_{6}^{(1)}, \mathbf{Z}_{1}^{(2)}, \mathbf{Z}_{2}^{(2)}, . ., \mathbf{Z}_{6}^{(2)}, . ., \mathbf{Z}_{1}^{(8)}, \mathbf{Z}_{2}^{(8)}, . ., \mathbf{Z}_{6}^{(8)}, \mathbf{Z}_{1}^{(9)}, \mathbf{Z}_{2}^{(9)}, \mathbf{Z}_{3}^{(9)}, \mathbf{Z}_{4}^{(9)}$.

Then the key under the consideration is cyclically shifted to the left by 25 positions. The resulting key block is again partitioned into eight subblocks that are assigned to the next eight subblock keys. This process is repeated until all 52 subblock keys are derived.

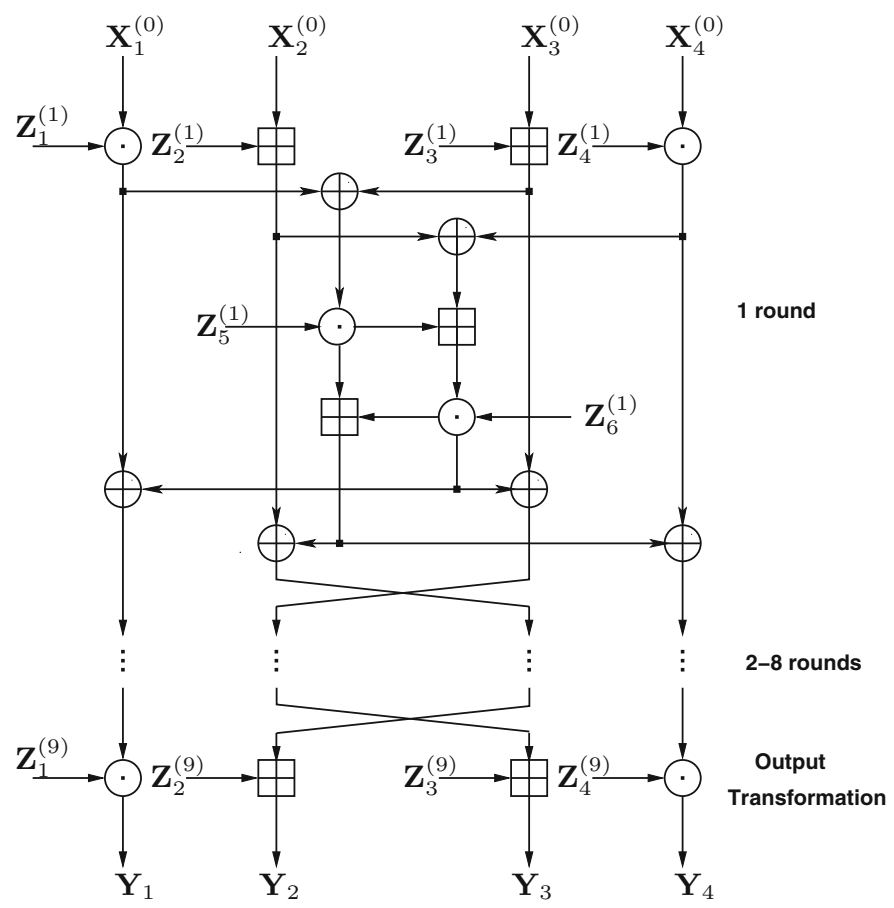

Fig. 1. Computational graph for the encryption process of the IDEA cipher 
Table 4. 128-bit IDEA master key bits indices starts from 0 and ends with 127 (indexed left to right). Range of indices of this key used for each of 52 subblock keys generated by the key scheduling algorithm

\begin{tabular}{r|l|l|l|l|l|l}
\hline$r$ & $\mathbf{Z}_{1}$ & $\mathbf{Z}_{2}$ & $\mathbf{Z}_{3}$ & $\mathbf{Z}_{4}$ & $\mathbf{Z}_{5}$ & $\mathbf{Z}_{6}$ \\
\hline 1 & $0-15$ & $16-31$ & $32-47$ & $48-63$ & $64-79$ & $80-95$ \\
\hline 2 & $96-111$ & $112-127$ & $25-40$ & $41-56$ & $57-72$ & $73-88$ \\
\hline 3 & $89-104$ & $105-120$ & $121-8$ & $9-24$ & $50-65$ & $66-81$ \\
\hline 4 & $82-97$ & $98-113$ & $114-1$ & $2-17$ & $18-33$ & $34-49$ \\
\hline 5 & $75-90$ & $91-106$ & $107-122$ & $123-10$ & $11-26$ & $27-42$ \\
\hline 6 & $43-58$ & $59-74$ & $100-115$ & $116-3$ & $4-19$ & $20-35$ \\
\hline 7 & $36-51$ & $52-67$ & $68-83$ & $84-99$ & $125-12$ & $13-28$ \\
\hline 8 & $29-44$ & $45-60$ & $61-76$ & $77-92$ & $93-108$ & $109-124$ \\
\hline 9 & $22-37$ & $38-53$ & $54-69$ & $70-85$ & - & - \\
\hline
\end{tabular}

\section{A.2 The MA-Structure and 1-Round IDEA Cipher}

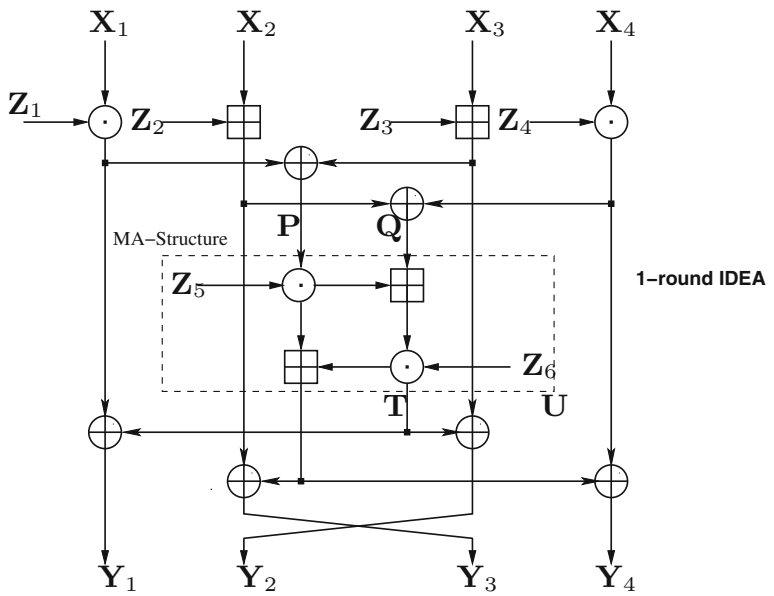

Fig. 2. Computational graph for the encryption process of 1-round IDEA cipher

Let us denote round key, input and output for the 1-round IDEA block cipher (see Fig. 2) as $Z=\left(\mathbf{Z}_{1}, \ldots, \mathbf{Z}_{6}\right), X=\left(\mathbf{X}_{1}, \mathbf{X}_{2}, \mathbf{X}_{3}, \mathbf{X}_{4}\right)$ and $Y=\left(\mathbf{Y}_{1}, \mathbf{Y}_{2}, \mathbf{Y}_{3}, \mathbf{Y}_{4}\right)$, where $\mathbf{Z}_{i}, \mathbf{X}_{i}, \mathbf{Y}_{i} \in \mathbb{Z}_{2}^{16}$, respectively. Then we have:

$$
\begin{aligned}
\mathbf{Y}_{1} & =\left(\mathbf{X}_{1} \odot \mathbf{Z}_{1}\right) \oplus \mathbf{T} . & \mathbf{Y}_{2} & =\left(\mathbf{X}_{3} \boxplus \mathbf{Z}_{3}\right) \oplus \mathbf{T} . \\
\mathbf{Y}_{3} & =\left(\mathbf{X}_{2} \boxplus \mathbf{Z}_{2}\right) \oplus \mathbf{U} . & \mathbf{Y}_{4} & =\left(\mathbf{X}_{4} \odot \mathbf{Z}_{4}\right) \oplus \mathbf{U} .
\end{aligned}
$$


We have the following equations for two input subblocks of the MA-structure $\mathbf{P}$ and $\mathbf{Q}$ and two output subblocks of the MA-structure $\mathbf{U}$ and $\mathbf{T}$ (see Fig. 2):

$$
\begin{array}{r}
\mathbf{P}=\left(\mathbf{X}_{1} \odot \mathbf{Z}_{1}\right) \oplus\left(\mathbf{X}_{3} \boxplus \mathbf{Z}_{3}\right) \text { and } \mathbf{Q}=\left(\mathbf{X}_{2} \boxplus \mathbf{Z}_{2}\right) \oplus\left(\mathbf{X}_{4} \odot \mathbf{Z}_{4}\right) . \\
\mathbf{U}=\left(\mathbf{P} \odot \mathbf{Z}_{5}\right) \boxplus \mathbf{T} \text { and } \mathbf{T}=\left[\left(\mathbf{P} \odot \mathbf{Z}_{5}\right) \boxplus \mathbf{Q}\right] \odot \mathbf{Z}_{6} .
\end{array}
$$

It is easy to see that $\mathbf{Y}_{1} \oplus \mathbf{Y}_{2}=\mathbf{P}$ and $\mathbf{Y}_{3} \oplus \mathbf{Y}_{4}=\mathbf{Q}$.

\section{B Appendix: New Linear Relations for 1-Round IDEA and 8.5-Round IDEA}

Table 5. List of new linear relations for 1-round IDEA, based on linear relations of Table 1, generated by Algorithm 1 . Here $\mathrm{k}$ is a non-negative integer, $-1 \equiv 0 \bmod$

\begin{tabular}{|c|c|c|c|c|c|c|c|c|c|c|}
\hline$\phi$ & $\psi$ & $\omega$ & $\lambda$ & $z_{1}$ & $z_{2}$ & $z_{3}$ & $z_{4}$ & $z_{5}$ & $z_{6}$ & \# of free bits \\
\hline $55(\mathbf{1}, \mathbf{2}, \mathbf{2}, \mathbf{1}, \mathbf{0}, \mathbf{0})$ & $(1,2,2,1)$ & $(\mathbf{3}, \mathbf{3}, \mathbf{3}, \mathbf{3})$ & 0 & $\mp 2$ & $2 k+1$ & $2 k+1$ & $\mp 2$ & - & - & 62 \\
\hline $56(\mathbf{0}, \mathbf{1}, \mathbf{0}, \mathbf{1}, \mathbf{1}, \mathbf{1})$ & $(\mathbf{0}, \mathbf{1}, \mathbf{1}, \mathbf{1})$ & $(3,2,0,0)$ & 1 & - & - & $2 k$ & $\mp 1$ & $\mp 2^{15}$ & $\mp 1$ & 50 \\
\hline $57(\mathbf{1}, \mathbf{1}, \mathbf{2}, \mathbf{1}, \mathbf{1}, \mathbf{1})$ & $(\mathbf{1}, \mathbf{1}, \mathbf{3}, \mathbf{1})$ & $(\mathbf{0}, \mathbf{3}, \mathbf{0}, \mathbf{0})$ & 1 & $\mp 1$ & - & $2 k+1$ & $\mp 1$ & $\mp 1$ & $\mp 2$ & 34 \\
\hline $58(\mathbf{0}, \mathbf{1}, \mathbf{3}, \mathbf{1}, \mathbf{1}, \mathbf{1})$ & $(\mathbf{0}, \mathbf{1}, \mathbf{3}, \mathbf{1})$ & $(1,2,0,0)$ & 0 & - & - & $2 k$ & $\mp 1$ & $\mp 1$ & $\mp 2$ & 49 \\
\hline $59(\mathbf{1}, \mathbf{1}, \mathbf{1}, \mathbf{1}, \mathbf{1}, \mathbf{1})$ & $(\mathbf{3}, \mathbf{1}, \mathbf{0}, \mathbf{3})$ & $(\mathbf{2}, \mathbf{3}, \mathbf{0}, \mathbf{0})$ & 0 & $\mp 2^{15}$ & - & $2 k$ & $\mp 2^{15}$ & $\mp 2^{15}$ & $\mp 1$ & 35 \\
\hline $60(\mathbf{1}, \mathbf{3}, \mathbf{0}, \mathbf{1}, \mathbf{0}, \mathbf{1})$ & $(\mathbf{1}, \mathbf{3}, \mathbf{1}, \mathbf{1})$ & $(3, \mathbf{1}, \mathbf{3}, \mathbf{2})$ & 0 & $\mp 2$ & $2 k+1$ & $2 k+1$ & $\mp 2$ & - & $\mp 2$ & 46 \\
\hline $61(\mathbf{0}, \mathbf{0}, \mathbf{0}, \mathbf{0}, \mathbf{1}, \mathbf{1})$ & $(\mathbf{0}, \mathbf{0}, \mathbf{1}, \mathbf{0})$ & $(3,2,1,1)$ & 1 & - & - & $2 k+1$ & - & $\mp 2^{15}$ & $\mp 1$ & 65 \\
\hline $62(\mathbf{1}, \mathbf{1}, \mathbf{1}, \mathbf{1}, \mathbf{1}, \mathbf{1})$ & $(\mathbf{1}, \mathbf{1}, \mathbf{0}, \mathbf{1})$ & $(2, \mathbf{1}, \mathbf{0}, \mathbf{0})$ & 1 & $\mp 2$ & - & $2 k+1$ & $\mp 1$ & $\mp 1$ & $\mp 2$ & 33 \\
\hline $63(\mathbf{1}, \mathbf{0}, \mathbf{3}, \mathbf{0}, \mathbf{1}, \mathbf{1})$ & $(3, \mathbf{0}, \mathbf{2}, \mathbf{0})$ & $(2,1,1,1)$ & 0 & $\mp 2^{15}$ & - & $2 k$ & - & $\mp 2^{15}$ & $\mp 2$ & 49 \\
\hline $64(\mathbf{0}, \mathbf{1}, \mathbf{2}, \mathbf{1}, \mathbf{1}, \mathbf{1})$ & $(\mathbf{0}, \mathbf{1}, \mathbf{2}, \mathbf{1})$ & $(\mathbf{3}, \mathbf{0}, \mathbf{0}, \mathbf{0})$ & 1 & - & - & $2 k+1$ & $\mp 1$ & $\mp 2^{15}$ & $\mp 2$ & 49 \\
\hline $65(\mathbf{1}, \mathbf{2}, \mathbf{3}, \mathbf{1}, \mathbf{1}, \mathbf{1})$ & $(\mathbf{3}, \mathbf{2}, \mathbf{3}, \mathbf{1})$ & $(2,1,2,2)$ & 0 & $\mp 2^{15}$ & $2 k+1$ & $2 k+1$ & $\mp 2$ & $\mp 2^{15}$ & $\mp 2$ & 32 \\
\hline $66(\mathbf{1}, \mathbf{2}, \mathbf{2}, \mathbf{1}, \mathbf{0}, \mathbf{0})$ & $(\mathbf{1}, \mathbf{3}, \mathbf{2}, \mathbf{1})$ & $(\mathbf{3}, \mathbf{3}, \mathbf{3}, \mathbf{3})$ & 1 & $\mp 2$ & $2 k$ & $2 k+1$ & $\mp 2$ & - & - & 62 \\
\hline $67(\mathbf{1}, \mathbf{2}, \mathbf{3}, \mathbf{1}, \mathbf{1}, \mathbf{1})$ & $(\mathbf{1}, \mathbf{2}, \mathbf{2}, \mathbf{1})$ & $(2,3,2,2)$ & 1 & $\mp 2$ & $2 k+1$ & $2 k$ & $\mp 2$ & $\mp 1$ & $\mp 1$ & 32 \\
\hline $68(\mathbf{1}, \mathbf{2}, \mathbf{3}, \mathbf{1}, \mathbf{1}, \mathbf{1})$ & $(\mathbf{1}, \mathbf{3}, \mathbf{2}, \mathbf{1})$ & $(2,3,2,2)$ & 0 & $\mp 2$ & $2 k$ & $2 k$ & $\mp 2$ & $\mp 1$ & $\mp 1$ & 32 \\
\hline $69(\mathbf{0}, \mathbf{0}, \mathbf{2}, \mathbf{1}, \mathbf{0}, \mathbf{1})$ & $(\mathbf{0}, \mathbf{0}, \mathbf{3}, \mathbf{1})$ & $(\mathbf{0}, \mathbf{2}, \mathbf{1}, \mathbf{0})$ & 1 . & - & - & $2 k+1$ & $\mp 1$ & - & $\mp 2$ & 64 \\
\hline $70(\mathbf{0}, \mathbf{0}, \mathbf{0}, \mathbf{1}, \mathbf{1}, \mathbf{0})$ & $(\mathbf{0}, \mathbf{0}, \mathbf{1}, \mathbf{1})$ & $(\mathbf{3}, \mathbf{2}, \mathbf{0}, \mathbf{1})$ & 1 & - & - & $2 k+1$ & $\mp 1$ & $\mp 2^{15}$ & - & 65 \\
\hline $71(\mathbf{1}, \mathbf{0}, \mathbf{3}, \mathbf{1}, \mathbf{0}, \mathbf{1})$ & $(\mathbf{3}, \mathbf{0}, \mathbf{3}, \mathbf{3})$ & $(\mathbf{1}, \mathbf{3}, \mathbf{1}, \mathbf{0})$ & 0 & $\mp 2^{15}$ & - & $2 k$ & $\mp 2^{15}$ & - & $\mp 2$ & 49 \\
\hline $72(\mathbf{1}, \mathbf{0}, \mathbf{3}, \mathbf{1}, \mathbf{1}, \mathbf{0})$ & $(\mathbf{1}, \mathbf{0}, \mathbf{2}, \mathbf{3})$ & $(\mathbf{2}, \mathbf{3}, \mathbf{0}, \mathbf{1})$ & 0 & $\mp 2$ & - & $2 k$ & $\mp 2^{15}$ & $\mp 1$ & - & 49 \\
\hline $73(\mathbf{1}, \mathbf{1}, \mathbf{3}, \mathbf{1}, \mathbf{1}, \mathbf{1})$ & $(\mathbf{1}, \mathbf{1}, \mathbf{3}, \mathbf{1})$ & $(2, \mathbf{1}, \mathbf{0}, \mathbf{0})$ & 0 & $\mp 1$ & - & $2 k+1$ & $\mp 1$ & $\mp 2^{15}$ & $\mp 2$ & 34 \\
\hline $74(\mathbf{1}, \mathbf{2}, \mathbf{1}, \mathbf{1}, \mathbf{1}, \mathbf{1})$ & $(3,2,0,1)$ & $(2,3,2,2)$ & 1 & $\mp 2^{15}$ & $2 k+1$ & $2 k+1$ & $\mp 2$ & $\mp 2^{15}$ & $\mp 1$ & 33 \\
\hline $75(\mathbf{1}, \mathbf{2}, \mathbf{1}, \mathbf{1}, \mathbf{1}, \mathbf{1})$ & $(\mathbf{3}, \mathbf{3}, \mathbf{0}, \mathbf{1})$ & $(2,3,2,2)$ & 0 & $\mp 2^{15}$ & $2 k$ & $2 k+1$ & $\mp 2$ & $\mp 2^{15}$ & $\mp 1$ & 33 \\
\hline $76(\mathbf{1}, \mathbf{2}, \mathbf{3}, \mathbf{1}, \mathbf{1}, \mathbf{1})$ & $(\mathbf{3}, \mathbf{3}, \mathbf{3}, \mathbf{1})$ & $(2,1,2,2)$ & 1 & $\mp 2^{15}$ & $2 k$ & $2 k+1$ & $\mp 2$ & $\mp 2^{15}$ & $\mp 2$ & 32 \\
\hline $77(\mathbf{1}, \mathbf{0}, \mathbf{1}, \mathbf{1}, \mathbf{1}, \mathbf{0})$ & $(\mathbf{3}, \mathbf{0}, \mathbf{0}, \mathbf{3})$ & $(\mathbf{2}, \mathbf{3}, \mathbf{0}, \mathbf{1})$ & 0 & $\mp 2^{15}$ & - & $2 k+1$ & $\mp 2^{15}$ & $\mp 2^{15}$ & - & 50 \\
\hline $78(\mathbf{0}, \mathbf{1}, \mathbf{2}, \mathbf{1}, \mathbf{1}, \mathbf{1})$ & $(\mathbf{0}, \mathbf{1}, \mathbf{3}, \mathbf{3})$ & $(\mathbf{3}, \mathbf{0}, \mathbf{0}, \mathbf{0})$ & 0 & - & - & $2 k$ & $\mp 2^{15}$ & $\mp 2^{15}$ & $\mp 2$ & 49 \\
\hline
\end{tabular}
$\left(2^{16}+1\right),-2^{15} \equiv 2^{15}+1 \bmod \left(2^{16}+1\right)$ and $-2 \equiv 2^{16}-1 \bmod \left(2^{16}+1\right)$. 
Table 5. (continued)

\begin{tabular}{|c|c|c|c|c|c|c|c|c|c|c|c|}
\hline & $\phi$ & $\psi$ & $\omega$ & & $z_{1}$ & $z_{2}$ & $z_{3}$ & $z_{4}$ & $z_{5}$ & $z_{6}$ & \# of free bits \\
\hline 79 & $(1,1,2,1,1,1)$ & $(\mathbf{1}, \mathbf{1}, \mathbf{2}, \mathbf{3})$ & $(\mathbf{0}, \mathbf{3}, \mathbf{0}, \mathbf{0})$ & 1 & $\mp 1$ & - & $2 k$ & $\mp 2^{15}$ & $\mp 1$ & $\mp 2$ & 34 \\
\hline 80 & $(\mathbf{1}, \mathbf{1}, \mathbf{1}, \mathbf{1}, \mathbf{1}, \mathbf{1})$ & $(\mathbf{1}, \mathbf{1}, \mathbf{0}, \mathbf{1})$ & $(2,3,0,0)$ & 1 & $\mp 1$ & - & $2 k+1$ & $\mp 1$ & $\mp 2^{15}$ & $\mp 1$ & 35 \\
\hline 81 & $(\mathbf{1}, \mathbf{3}, \mathbf{1}, \mathbf{1}, \mathbf{1}, \mathbf{0})$ & $(\mathbf{1}, \mathbf{3}, \mathbf{0}, \mathbf{1})$ & $(2,3,2,3)$ & 1 & $\mp 1$ & $2 k+1$ & $2 k$ & $\mp 2$ & $\mp 2^{15}$ & - & 48 \\
\hline 82 & $(\mathbf{1}, \mathbf{3}, \mathbf{3}, \mathbf{1}, \mathbf{1}, \mathbf{0})$ & $(\mathbf{1}, \mathbf{2}, \mathbf{3}, \mathbf{1})$ & $(2,3,2,3)$ & 0 & $\mp 2$ & $2 k$ & $2 k+1$ & $\mp 2$ & $\mp 1$ & - & 47 \\
\hline 83 & $(\mathbf{1}, \mathbf{0}, \mathbf{1}, \mathbf{0}, \mathbf{1}, \mathbf{1})$ & $(\mathbf{1}, \mathbf{0}, \mathbf{0}, \mathbf{0})$ & $(\mathbf{2}, \mathbf{1}, \mathbf{1}, \mathbf{1})$ & 1 & $\mp 2$ & - & $2 k$ & - & $\mp 1$ & $\mp 2$ & 48 \\
\hline 84 & $(\mathbf{1}, \mathbf{1}, \mathbf{3}, \mathbf{0}, \mathbf{1}, \mathbf{0})$ & $(\mathbf{1}, \mathbf{1}, \mathbf{2}, \mathbf{0})$ & $(2,3, \mathbf{1}, \mathbf{0})$ & 1 & $\mp 2$ & - & $2 k$ & - & $\mp 1$ & - & 64 \\
\hline 85 & $(1,2,2,1,1,1)$ & $(\mathbf{1}, \mathbf{3}, \mathbf{3}, \mathbf{1})$ & $(\mathbf{0}, \mathbf{1}, \mathbf{2}, \mathbf{2})$ & 0 & $\mp 2$ & $2 k$ & $2 k+1$ & $\mp 2$ & $\mp 2^{15}$ & $\mp 1$ & 32 \\
\hline 86 & $(\mathbf{1}, \mathbf{0}, \mathbf{0}, \mathbf{1}, \mathbf{0}, \mathbf{1})$ & $(\mathbf{1}, \mathbf{0}, \mathbf{1}, \mathbf{1})$ & $(\mathbf{3}, \mathbf{1}, \mathbf{1}, \mathbf{0})$ & 0 & $\mp 2$ & - & $2 k+1$ & $\mp 1$ & - & $\mp 2$ & 48 \\
\hline 87 & $(\mathbf{0}, \mathbf{0}, \mathbf{2}, \mathbf{1}, \mathbf{0}, \mathbf{1})$ & $(\mathbf{0}, \mathbf{0}, \mathbf{2}, \mathbf{3})$ & $(\mathbf{0}, \mathbf{2}, \mathbf{1}, \mathbf{0})$ & 1 & - & - & $2 k$ & $\mp 2^{15}$ & - & $\mp 2$ & 64 \\
\hline 88 & $(\mathbf{1}, \mathbf{1}, \mathbf{2}, \mathbf{1}, \mathbf{1}, \mathbf{1})$ & $(\mathbf{3}, \mathbf{1}, \mathbf{3}, \mathbf{1})$ & $(\mathbf{0}, \mathbf{3}, \mathbf{0}, \mathbf{0})$ & 1 & $\mp 2^{15}$ & - & $2 k+1$ & $\mp 1$ & $\mp 1$ & $\mp 2$ & 34 \\
\hline 89 & $(\mathbf{1}, \mathbf{3}, \mathbf{0}, \mathbf{1}, \mathbf{1}, \mathbf{0})$ & $(\mathbf{1}, \mathbf{3}, \mathbf{0}, \mathbf{1})$ & $(\mathbf{0}, \mathbf{1}, \mathbf{2}, \mathbf{3})$ & 1 & $\mp 1$ & $2 k+1$ & - & $\mp 2$ & $\mp 1$ & - & 49 \\
\hline 90 & $(\mathbf{1}, \mathbf{3}, \mathbf{3}, \mathbf{1}, \mathbf{0}, \mathbf{1})$ & $(1,2,2,1)$ & $(\mathbf{1}, \mathbf{3}, \mathbf{3}, \mathbf{2})$ & 1 & $\mp 1$ & $2 k$ & $2 k+1$ & $\mp 2$ & - & $\mp 2$ & 47 \\
\hline 91 & $(\mathbf{1}, \mathbf{1}, \mathbf{1}, \mathbf{0}, \mathbf{1}, \mathbf{0})$ & $(\mathbf{3}, \mathbf{1}, \mathbf{0}, \mathbf{0})$ & $(2,3,1,0)$ & 1 & $\mp 2^{15}$ & - & $2 k+1$ & - & $\mp 2^{15}$ & - & 65 \\
\hline 92 & $(\mathbf{1}, \mathbf{1}, \mathbf{2}, \mathbf{1}, \mathbf{0}, \mathbf{0})$ & $(\mathbf{1}, \mathbf{1}, \mathbf{2}, \mathbf{1})$ & $(\mathbf{3}, \mathbf{3}, \mathbf{1}, \mathbf{1})$ & 0 & $\mp 2$ & - & $2 k+1$ & $\mp 1$ & - & - & 64 \\
\hline 93 & $(\mathbf{1}, \mathbf{2}, \mathbf{3}, \mathbf{1}, \mathbf{1}, \mathbf{1})$ & $(1,2,2,1)$ & $(2,1,2,2)$ & 1 & $\mp 1$ & $2 k+1$ & $2 k$ & $\mp 2$ & $\mp 2^{15}$ & $\mp 2$ & 32 \\
\hline 94 & $(\mathbf{0}, \mathbf{1}, \mathbf{3}, \mathbf{1}, \mathbf{1}, \mathbf{1})$ & $(\mathbf{0}, \mathbf{1}, \mathbf{2}, \mathbf{3})$ & $(\mathbf{1}, \mathbf{2}, \mathbf{0}, \mathbf{0})$ & 0 & - & - & $2 k+1$ & $\mp 2^{15}$ & $\mp 1$ & $\mp 2$ & 49 \\
\hline 95 & $(\mathbf{1}, \mathbf{2}, \mathbf{3}, \mathbf{1}, \mathbf{1}, \mathbf{1})$ & $(\mathbf{1}, \mathbf{3}, \mathbf{2}, \mathbf{1})$ & $(2,1,2,2)$ & 0 & $\mp 1$ & $2 k$ & $2 k$ & $\mp 2$ & $\mp 2^{15}$ & $\mp 2$ & 32 \\
\hline 96 & $(\mathbf{1}, \mathbf{1}, \mathbf{3}, \mathbf{1}, \mathbf{1}, \mathbf{1})$ & $(\mathbf{1}, \mathbf{1}, \mathbf{3}, \mathbf{3})$ & $(\mathbf{2}, \mathbf{3}, \mathbf{0}, \mathbf{0})$ & 0 & $\mp 2$ & - & $2 k+1$ & $\mp 2^{15}$ & $\mp 1$ & $\mp 1$ & 34 \\
\hline 97 & $(\mathbf{1}, \mathbf{0}, \mathbf{1}, \mathbf{0}, \mathbf{1}, \mathbf{1})$ & $(\mathbf{1}, \mathbf{0}, \mathbf{0}, \mathbf{0})$ & $(2,3,1,1)$ & 1 & $\mp 1$ & - & $2 k$ & - & $\mp 2^{15}$ & $\mp 1$ & 50 \\
\hline 98 & $(\mathbf{0}, \mathbf{2}, \mathbf{0}, \mathbf{1}, \mathbf{0}, \mathbf{0})$ & $(\mathbf{0}, \mathbf{2}, \mathbf{0}, \mathbf{1})$ & $(\mathbf{0}, \mathbf{0}, \mathbf{3}, \mathbf{3})$ & 1 & - & $2 k+1$ & - & $\mp 2$ & - & - & 79 \\
\hline 99 & $(\mathbf{0}, \mathbf{3}, \mathbf{1}, \mathbf{1}, \mathbf{1}, \mathbf{0})$ & $(\mathbf{0}, \mathbf{2}, \mathbf{1}, \mathbf{1})$ & $(\mathbf{1}, \mathbf{0}, \mathbf{2}, \mathbf{3})$ & 1 & - & $2 k$ & - & $\mp 2$ & $\mp 1$ & - & 64 \\
\hline 100 & $(\mathbf{1}, \mathbf{1}, \mathbf{3}, \mathbf{1}, \mathbf{1}, \mathbf{1})$ & $(\mathbf{3}, \mathbf{1}, \mathbf{3}, \mathbf{1})$ & $(2, \mathbf{1}, \mathbf{0}, \mathbf{0})$ & 0 & $\mp 2^{15}$ & - & $2 k+1$ & $\mp 1$ & $\mp 2^{15}$ & $\mp 2$ & 34 \\
\hline 101 & $(\mathbf{1}, \mathbf{3}, \mathbf{2}, \mathbf{1}, \mathbf{0}, \mathbf{1})$ & $(\mathbf{1}, \mathbf{3}, \mathbf{3}, \mathbf{1})$ & $(\mathbf{3}, \mathbf{3}, \mathbf{3}, \mathbf{2})$ & 0 & $\mp 2$ & $2 k+1$ & $2 k$ & $\mp 2$ & - & $\mp 1$ & 47 \\
\hline 102 & $(\mathbf{1}, \mathbf{1}, \mathbf{3}, \mathbf{1}, \mathbf{1}, \mathbf{1})$ & $(\mathbf{1}, \mathbf{1}, \mathbf{2}, \mathbf{1})$ & $(\mathbf{2}, \mathbf{3}, \mathbf{0}, \mathbf{0})$ & 1 & $\mp 2$ & - & $2 k$ & $\mp 1$ & $\mp 1$ & $\mp 1$ & 34 \\
\hline 103 & $(\mathbf{1}, \mathbf{3}, \mathbf{1}, \mathbf{1}, \mathbf{0}, \mathbf{1})$ & $(\mathbf{1}, \mathbf{2}, \mathbf{1}, \mathbf{1})$ & $(\mathbf{1}, \mathbf{1}, \mathbf{3}, \mathbf{2})$ & 0 & $\mp 1$ & $2 k$ & - & $\mp 2$ & - & $\mp 1$ & 49 \\
\hline 104 & $(\mathbf{1}, \mathbf{1}, \mathbf{2}, \mathbf{0}, \mathbf{1}, \mathbf{0})$ & $(\mathbf{1}, \mathbf{1}, \mathbf{3}, \mathbf{0})$ & $(\mathbf{0}, \mathbf{1}, \mathbf{1}, \mathbf{0})$ & 1 & $\mp 2$ & - & $2 k+1$ & - & $\mp 2^{15}$ & - & 64 \\
\hline 105 & $(\mathbf{1}, \mathbf{1}, \mathbf{3}, \mathbf{0}, \mathbf{0}, \mathbf{1})$ & $(\mathbf{1}, \mathbf{1}, \mathbf{2}, \mathbf{0})$ & $(\mathbf{1}, \mathbf{3}, \mathbf{0}, \mathbf{1})$ & 1 & $\mp 1$ & - & $2 k+1$ & - & - & $\mp 2$ & 64 \\
\hline 106 & $(\mathbf{1}, \mathbf{0}, \mathbf{1}, \mathbf{1}, \mathbf{1}, \mathbf{0})$ & $(\mathbf{1}, \mathbf{0}, \mathbf{0}, \mathbf{1})$ & $(\mathbf{2}, \mathbf{3}, \mathbf{0}, \mathbf{1})$ & 1 & $\mp 1$ & - & $2 k$ & $\mp 1$ & $\mp 2^{15}$ & - & 50 \\
\hline 107 & $(\mathbf{1}, \mathbf{1}, \mathbf{2}, \mathbf{1}, \mathbf{1}, \mathbf{1})$ & $(\mathbf{3}, \mathbf{1}, \mathbf{2}, \mathbf{3})$ & $(\mathbf{0}, \mathbf{3}, \mathbf{0}, \mathbf{0})$ & 1 & $\mp 2^{15}$ & - & $2 k$ & $\mp 2^{15}$ & $\mp 1$ & $\mp 2$ & 34 \\
\hline 108 & $(\mathbf{1}, \mathbf{3}, \mathbf{2}, \mathbf{1}, \mathbf{0}, \mathbf{1})$ & $(\mathbf{1}, 2,2,1)$ & $(\mathbf{3}, \mathbf{3}, \mathbf{3}, \mathbf{2})$ & 0 & $\mp 2$ & $2 k$ & $2 k+1$ & $\mp 2$ & - & $\mp 1$ & 47 \\
\hline 109 & $(\mathbf{1}, \mathbf{1}, \mathbf{1}, \mathbf{1}, \mathbf{1}, \mathbf{1})$ & $(\mathbf{3}, \mathbf{1}, \mathbf{0}, \mathbf{1})$ & $(\mathbf{2}, \mathbf{3}, \mathbf{0}, \mathbf{0})$ & 1 & $\mp 2^{15}$ & - & $2 k+1$ & $\mp 1$ & $\mp 2^{15}$ & $\mp 1$ & 35 \\
\hline 110 & $(\mathbf{1}, \mathbf{2}, \mathbf{1}, \mathbf{1}, \mathbf{0}, \mathbf{0})$ & $(\mathbf{3}, \mathbf{2}, \mathbf{1}, \mathbf{1})$ & $(\mathbf{1}, \mathbf{1}, \mathbf{3}, \mathbf{3})$ & 0 & $\mp 2^{15}$ & $2 k+1$ & - & $\mp 2$ & - & - & 64 \\
\hline 111 & $(1,3,1,1,1,0)$ & $(\mathbf{3}, \mathbf{3}, \mathbf{0}, \mathbf{1})$ & $(2,3,2,3)$ & 1 & $\mp 2^{15}$ & $2 k+1$ & $2 k$ & $\mp 2$ & $\mp 2^{15}$ & - & 48 \\
\hline 112 & $(\mathbf{1}, \mathbf{3}, \mathbf{0}, \mathbf{1}, \mathbf{0}, \mathbf{1})$ & $(\mathbf{1}, \mathbf{2}, \mathbf{1}, \mathbf{1})$ & $(\mathbf{3}, \mathbf{1}, \mathbf{3}, \mathbf{2})$ & 0 & $\mp 2$ & $2 k$ & $2 k$ & $\mp 2$ & - & $\mp 2$ & 46 \\
\hline 113 & $(\mathbf{1}, \mathbf{3}, \mathbf{1}, \mathbf{1}, \mathbf{1}, \mathbf{0})$ & $(\mathbf{1}, \mathbf{2}, \mathbf{0}, \mathbf{1})$ & $(\mathbf{2}, \mathbf{3}, \mathbf{2}, \mathbf{3})$ & 1 & $\mp 1$ & $2 k$ & $2 k+1$ & $\mp 2$ & $\mp 2^{15}$ & - & 48 \\
\hline 114 & $(1,2,2,1,1,1)$ & $(\mathbf{1}, \mathbf{2}, \mathbf{3}, \mathbf{1})$ & $(\mathbf{0}, \mathbf{1}, \mathbf{2}, \mathbf{2})$ & 1 & $\mp 2$ & $2 k+1$ & $2 k+1$ & $\mp 2$ & $\mp 2^{15}$ & $\mp 1$ & 32 \\
\hline 115 & $(\mathbf{1}, \mathbf{0}, \mathbf{2}, \mathbf{1}, \mathbf{1}, \mathbf{0})$ & $(\mathbf{1}, \mathbf{0}, \mathbf{3}, \mathbf{3})$ & $(\mathbf{0}, \mathbf{1}, \mathbf{0}, \mathbf{1})$ & 0 & $\mp 2$ & - & $2 k+1$ & $\mp 2^{15}$ & $\mp 2^{15}$ & - & 49 \\
\hline 116 & $(\mathbf{1}, \mathbf{3}, \mathbf{3}, \mathbf{1}, \mathbf{0}, \mathbf{1})$ & $(3,2,2,1)$ & $(\mathbf{1}, \mathbf{3}, \mathbf{3}, \mathbf{2})$ & 1 & $\mp 2^{15}$ & $2 k$ & $2 k+1$ & $\mp 2$ & - & $\mp 2$ & 47 \\
\hline 117 & $(0,2,0,1,1,1)$ & $(\mathbf{0}, \mathbf{3}, \mathbf{1}, \mathbf{1})$ & $(3,2,2,2)$ & 1 & - & $2 k$ & $2 k+1$ & $\mp 2$ & $\mp 2^{15}$ & $\mp 1$ & 48 \\
\hline
\end{tabular}


Table 5. (continued)

\begin{tabular}{|c|c|c|c|c|c|c|c|c|c|c|c|}
\hline & $\phi$ & $\psi$ & $\omega$ & $\lambda$ & $z_{1}$ & $z_{2}$ & $z_{3}$ & $z_{4}$ & $z_{5}$ & $z_{6}$ & \# of free bits \\
\hline 118 & $(\mathbf{0}, \mathbf{3}, \mathbf{0}, \mathbf{1}, \mathbf{0}, \mathbf{1})$ & $(\mathbf{0}, \mathbf{2}, \mathbf{0}, \mathbf{1})$ & $(\mathbf{0}, \mathbf{0}, \mathbf{3}, \mathbf{2})$ & 1 & - & $2 k$ & - & $\mp 2$ & - & $\mp 1$ & 64 \\
\hline 119 & $(\mathbf{1}, \mathbf{1}, \mathbf{2}, \mathbf{0}, \mathbf{0}, \mathbf{1})$ & $(\mathbf{1}, \mathbf{1}, \mathbf{2}, \mathbf{0})$ & $(\mathbf{3}, \mathbf{3}, \mathbf{0}, \mathbf{1})$ & 0 & $\mp 2$ & - & $2 k+1$ & - & - & $\mp 1$ & 64 \\
\hline 120 & $(\mathbf{0}, \mathbf{3}, \mathbf{0}, \mathbf{1}, \mathbf{1}, \mathbf{0})$ & $(\mathbf{0}, \mathbf{2}, \mathbf{1}, \mathbf{1})$ & $(\mathbf{3}, \mathbf{2}, \mathbf{2}, \mathbf{3})$ & 1 . & - & $2 k$ & $2 k$ & $\mp 2$ & $\mp 2^{15}$ & - & 63 \\
\hline 121 & $(\mathbf{1}, \mathbf{2}, \mathbf{3}, \mathbf{1}, \mathbf{1}, \mathbf{1})$ & $(\mathbf{3}, \mathbf{3}, \mathbf{2}, \mathbf{1})$ & $(\mathbf{2}, \mathbf{1}, \mathbf{2}, \mathbf{2})$ & 0 & $\mp 2^{15}$ & $2 k$ & $2 k$ & $\mp 2$ & $\mp 2^{15}$ & $\mp 2$ & 32 \\
\hline 122 & $(\mathbf{0}, \mathbf{0}, \mathbf{2}, \mathbf{1}, \mathbf{0}, \mathbf{1})$ & $(\mathbf{0}, \mathbf{0}, \mathbf{3}, \mathbf{3})$ & $(\mathbf{0}, \mathbf{2}, \mathbf{1}, \mathbf{0})$ & 1 & - & - & $2 k+1$ & $\mp 2^{15}$ & - & $\mp 2$ & 64 \\
\hline 123 & $(\mathbf{1}, \mathbf{1}, \mathbf{3}, \mathbf{1}, \mathbf{1}, \mathbf{1})$ & $(\mathbf{1}, \mathbf{1}, \mathbf{3}, \mathbf{3})$ & $(2, \mathbf{1}, \mathbf{0}, \mathbf{0})$ & 0 & $\mp 1$ & - & $2 k+1$ & $\mp 2^{15}$ & $\mp 2^{15}$ & $\mp 2$ & 34 \\
\hline 124 & $(\mathbf{1}, \mathbf{1}, \mathbf{0}, \mathbf{0}, \mathbf{0}, \mathbf{1})$ & $(\mathbf{1}, \mathbf{1}, \mathbf{1}, \mathbf{0})$ & $(\mathbf{3}, \mathbf{1}, \mathbf{0}, \mathbf{1})$ & 0 & $\mp 2$ & - & $2 k$ & - & - & $\mp 2$ & 63 \\
\hline 125 & $(\mathbf{0}, \mathbf{1}, \mathbf{0}, \mathbf{0}, \mathbf{1}, \mathbf{0})$ & $(\mathbf{0}, \mathbf{1}, \mathbf{1}, \mathbf{0})$ & $(\mathbf{3}, \mathbf{2}, \mathbf{1}, \mathbf{0})$ & 0 & - & - & $2 k+1$ & - & $\mp 2^{15}$ & - & 80 \\
\hline 126 & $(\mathbf{1}, \mathbf{0}, \mathbf{1}, \mathbf{0}, \mathbf{1}, \mathbf{1})$ & $(\mathbf{3}, \mathbf{0}, \mathbf{0}, \mathbf{0})$ & $(2, \mathbf{3}, \mathbf{1}, \mathbf{1})$ & 1 & $\mp 2^{15}$ & - & $2 k$ & - & $\mp 2^{15}$ & $\mp 1$ & 50 \\
\hline 127 & $(\mathbf{0}, \mathbf{0}, \mathbf{3}, \mathbf{0}, \mathbf{1}, \mathbf{1})$ & $(\mathbf{0}, \mathbf{0}, \mathbf{2}, \mathbf{0})$ & $(\mathbf{1}, \mathbf{2}, \mathbf{1}, \mathbf{1})$ & 1 & - & - & $2 k+1$ & - & $\mp 1$ & $\mp 2$ & 64 \\
\hline 128 & $(\mathbf{1}, \mathbf{3}, \mathbf{1}, \mathbf{1}, \mathbf{0}, \mathbf{1})$ & $(3,2,1,1)$ & $(\mathbf{1}, \mathbf{1}, \mathbf{3}, \mathbf{2})$ & 0 & $\mp 2^{15}$ & $2 k$ & - & $\mp 2$ & - & $\mp 1$ & 49 \\
\hline 129 & $(\mathbf{1}, \mathbf{2}, \mathbf{1}, \mathbf{1}, \mathbf{1}, \mathbf{1})$ & $(\mathbf{1}, \mathbf{2}, \mathbf{0}, \mathbf{1})$ & $(2,1,2,2)$ & 0 & $\mp 2$ & $2 k+1$ & $2 k$ & $\mp 2$ & $\mp 1$ & $\mp 2$ & 31 \\
\hline 130 & $(\mathbf{1}, \mathbf{1}, \mathbf{3}, \mathbf{0}, \mathbf{0}, \mathbf{1})$ & $(\mathbf{3}, \mathbf{1}, \mathbf{2}, \mathbf{0})$ & $(\mathbf{1}, \mathbf{3}, \mathbf{0}, \mathbf{1})$ & 1 & $\mp 2^{15}$ & - & $2 k+1$ & - & - & $\mp 2$ & 64 \\
\hline 131 & $(\mathbf{1}, \mathbf{0}, \mathbf{1}, \mathbf{1}, \mathbf{1}, \mathbf{0})$ & $(\mathbf{3}, \mathbf{0}, \mathbf{0}, \mathbf{1})$ & $(\mathbf{2}, \mathbf{3}, \mathbf{0}, \mathbf{1})$ & 1 & $\mp 2^{15}$ & - & $2 k$ & $\mp 1$ & $\mp 2^{15}$ & - & 50 \\
\hline 132 & $(\mathbf{1}, \mathbf{0}, \mathbf{2}, \mathbf{0}, \mathbf{1}, \mathbf{1})$ & $(\mathbf{1}, \mathbf{0}, \mathbf{2}, \mathbf{0})$ & $(\mathbf{0}, \mathbf{3}, \mathbf{1}, \mathbf{1})$ & 0 & $\mp 1$ & - & $2 k$ & - & $\mp 1$ & $\mp 2$ & 49 \\
\hline 133 & $(\mathbf{1}, \mathbf{0}, \mathbf{0}, \mathbf{0}, \mathbf{1}, \mathbf{1})$ & $(\mathbf{3}, \mathbf{0}, \mathbf{0}, \mathbf{0})$ & $(\mathbf{0}, \mathbf{1}, \mathbf{1}, \mathbf{1})$ & 1 & $\mp 2^{15}$ & - & - & - & $\mp 1$ & $\mp 1$ & 51 \\
\hline 134 & $(\mathbf{1}, \mathbf{3}, \mathbf{3}, \mathbf{1}, \mathbf{0}, \mathbf{1})$ & $(\mathbf{1}, \mathbf{2}, \mathbf{3}, \mathbf{1})$ & $(\mathbf{1}, \mathbf{3}, \mathbf{3}, \mathbf{2})$ & 1 & $\mp 1$ & $2 k$ & $2 k$ & $\mp 2$ & - & $\mp 2$ & 47 \\
\hline 135 & $(\mathbf{0}, \mathbf{1}, \mathbf{0}, \mathbf{1}, \mathbf{1}, \mathbf{1})$ & $(\mathbf{0}, \mathbf{1}, \mathbf{1}, \mathbf{3})$ & $(\mathbf{3}, \mathbf{2}, \mathbf{0}, \mathbf{0})$ & 1 & - & - & $2 k$ & $\mp 2^{15}$ & $\mp 2^{15}$ & $\mp 1$ & 50 \\
\hline 136 & $(\mathbf{1}, \mathbf{0}, \mathbf{0}, \mathbf{1}, \mathbf{0}, \mathbf{1})$ & $(\mathbf{1}, \mathbf{0}, \mathbf{1}, \mathbf{3})$ & $(\mathbf{3}, \mathbf{1}, \mathbf{1}, \mathbf{0})$ & 0 & $\mp 2$ & - & $2 k+1$ & $\mp 2^{15}$ & - & $\mp 2$ & 48 \\
\hline 137 & $(0,2,0,1,1,1)$ & $(0,2,1,1)$ & $(3,2,2,2)$ & 0 & - & $2 k+1$ & $2 k+1$ & $\mp 2$ & $\mp 2^{15}$ & $\mp 1$ & 48 \\
\hline 138 & $(\mathbf{1}, \mathbf{1}, \mathbf{2}, \mathbf{1}, \mathbf{1}, \mathbf{1})$ & $(\mathbf{1}, \mathbf{1}, \mathbf{3}, \mathbf{3})$ & $(\mathbf{0}, \mathbf{3}, \mathbf{0}, \mathbf{0})$ & 1 & $\mp 1$ & - & $2 k+1$ & $\mp 2^{15}$ & $\mp 1$ & $\mp 2$ & 34 \\
\hline 139 & $(\mathbf{1}, \mathbf{2}, \mathbf{1}, \mathbf{1}, \mathbf{1}, \mathbf{1})$ & $(\mathbf{1}, \mathbf{3}, \mathbf{0}, \mathbf{1})$ & $(2,1,2,2)$ & 1 & $\mp 2$ & $2 k$ & $2 k$ & $\mp 2$ & $\mp 1$ & $\mp 2$ & 31 \\
\hline 140 & $(\mathbf{0}, \mathbf{1}, \mathbf{3}, \mathbf{1}, \mathbf{1}, \mathbf{1})$ & $(\mathbf{0}, \mathbf{1}, \mathbf{3}, \mathbf{3})$ & $(\mathbf{1}, \mathbf{2}, \mathbf{0}, \mathbf{0})$ & 0 & - & - & $2 k$ & $\mp 2^{15}$ & $\mp 1$ & $\mp 2$ & 49 \\
\hline 141 & $(\mathbf{1}, \mathbf{3}, \mathbf{3}, \mathbf{1}, \mathbf{0}, \mathbf{1})$ & $(\mathbf{1}, \mathbf{3}, \mathbf{2}, \mathbf{1})$ & $(\mathbf{1}, \mathbf{3}, \mathbf{3}, \mathbf{2})$ & 0 & $\mp 1$ & $2 k+1$ & $2 k+1$ & $\mp 2$ & - & $\mp 2$ & 47 \\
\hline 142 & $(\mathbf{1}, \mathbf{1}, \mathbf{1}, \mathbf{1}, \mathbf{1}, \mathbf{1})$ & $(\mathbf{1}, \mathbf{1}, \mathbf{0}, \mathbf{3})$ & $(\mathbf{2}, \mathbf{1}, \mathbf{0}, \mathbf{0})$ & 1 & $\mp 2$ & - & $2 k+1$ & $\mp 2^{15}$ & $\mp 1$ & $\mp 2$ & 33 \\
\hline 143 & $(\mathbf{0}, \mathbf{1}, \mathbf{2}, \mathbf{1}, \mathbf{1}, \mathbf{1})$ & $(\mathbf{0}, \mathbf{1}, \mathbf{2}, \mathbf{3})$ & $(\mathbf{3}, \mathbf{0}, \mathbf{0}, \mathbf{0})$ & 1 & - & - & $2 k+1$ & $\mp 2^{15}$ & $\mp 2^{15}$ & $\mp 2$ & 49 \\
\hline 144 & $(\mathbf{1}, \mathbf{3}, \mathbf{3}, \mathbf{1}, \mathbf{1}, \mathbf{0})$ & $(\mathbf{1}, \mathbf{3}, \mathbf{3}, \mathbf{1})$ & $(2,3,2,3)$ & 1 & $\mp 2$ & $2 k+1$ & $2 k+1$ & $\mp 2$ & $\mp 1$ & - & 47 \\
\hline 145 & $(\mathbf{1}, \mathbf{2}, \mathbf{1}, \mathbf{1}, \mathbf{1}, \mathbf{1})$ & $(\mathbf{1}, \mathbf{2}, \mathbf{0}, \mathbf{1})$ & $(2,3,2,2)$ & 0 & $\mp 1$ & $2 k+1$ & $2 k$ & $\mp 2$ & $\mp 2^{15}$ & $\mp 1$ & 33 \\
\hline 146 & $(\mathbf{0}, \mathbf{3}, \mathbf{2}, \mathbf{1}, \mathbf{0}, \mathbf{1})$ & $(\mathbf{0}, \mathbf{2}, \mathbf{2}, \mathbf{1})$ & $(\mathbf{0}, \mathbf{2}, \mathbf{3}, \mathbf{2})$ & 0 & - & $2 k$ & $2 k$ & $\mp 2$ & - & $\mp 2$ & 62 \\
\hline 147 & $(\mathbf{1}, \mathbf{3}, \mathbf{0}, \mathbf{1}, \mathbf{1}, \mathbf{0})$ & $(\mathbf{3}, \mathbf{3}, \mathbf{0}, \mathbf{1})$ & $(\mathbf{0}, \mathbf{1}, \mathbf{2}, \mathbf{3})$ & 1 & $\mp 2^{15}$ & $2 k+1$ & - & $\mp 2$ & $\mp 1$ & - & 49 \\
\hline 148 & $(1,1,2,1,1,1)$ & $(\mathbf{1}, \mathbf{1}, \mathbf{3}, \mathbf{1})$ & $(\mathbf{0}, \mathbf{1}, \mathbf{0}, \mathbf{0})$ & 1 & $\mp 2$ & - & $2 k+1$ & $\mp 1$ & $\mp 2^{15}$ & $\mp 1$ & 34 \\
\hline 149 & $(1,2,3,1,1,1)$ & $(3,2,2,1)$ & $(2,1,2,2)$ & 1 & $\mp 2^{15}$ & $2 k+1$ & $2 k$ & $\mp 2$ & $\mp 2^{15}$ & $\mp 2$ & 32 \\
\hline 150 & $(\mathbf{0}, \mathbf{0}, \mathbf{0}, \mathbf{1}, \mathbf{1}, \mathbf{0})$ & $(\mathbf{0}, \mathbf{0}, \mathbf{1}, \mathbf{3})$ & $(3, \mathbf{2}, \mathbf{0}, \mathbf{1})$ & 1 & - & - & $2 k+1$ & $\mp 2^{15}$ & $\mp 2^{15}$ & - & 65 \\
\hline 151 & $(\mathbf{1}, \mathbf{3}, \mathbf{2}, \mathbf{1}, \mathbf{1}, \mathbf{0})$ & $(\mathbf{1}, \mathbf{2}, 2, \mathbf{1})$ & $(\mathbf{0}, \mathbf{1}, \mathbf{2}, \mathbf{3})$ & 1 & $\mp 2$ & $2 k$ & $2 k$ & $\mp 2$ & $\mp 2^{15}$ & - & 47 \\
\hline 152 & $(\mathbf{1}, \mathbf{1}, \mathbf{3}, \mathbf{1}, \mathbf{1}, \mathbf{1})$ & $(\mathbf{3}, \mathbf{1}, \mathbf{3}, \mathbf{3})$ & $(\mathbf{2}, \mathbf{1}, \mathbf{0}, \mathbf{0})$ & 0 & $\mp 2^{15}$ & - & $2 k+1$ & $\mp 2^{15}$ & $\mp 2^{15}$ & $\mp 2$ & 34 \\
\hline 153 & $(\mathbf{1}, \mathbf{1}, \mathbf{3}, \mathbf{0}, \mathbf{0}, \mathbf{1})$ & $(\mathbf{1}, \mathbf{1}, \mathbf{3}, \mathbf{0})$ & $(\mathbf{1}, \mathbf{3}, \mathbf{0}, \mathbf{1})$ & 1 & $\mp 1$ & - & $2 k$ & - & - & $\mp 2$ & 64 \\
\hline 154 & $(\mathbf{0}, \mathbf{3}, \mathbf{1}, \mathbf{1}, \mathbf{1}, \mathbf{0})$ & $(\mathbf{0}, \mathbf{3}, \mathbf{1}, \mathbf{1})$ & $(\mathbf{1}, \mathbf{0}, \mathbf{2}, \mathbf{3})$ & 0 & - & $2 k+1$ & - & $\mp 2$ & $\mp 1$ & - & 64 \\
\hline 155 & $(\mathbf{1}, \mathbf{1}, \mathbf{3}, \mathbf{1}, \mathbf{1}, \mathbf{1})$ & $(\mathbf{1}, \mathbf{1}, \mathbf{2}, \mathbf{1})$ & $(2, \mathbf{1}, \mathbf{0}, \mathbf{0})$ & 1 & $\mp 1$ & - & $2 k$ & $\mp 1$ & $\mp 2^{15}$ & $\mp 2$ & 34 \\
\hline 156 & $(\mathbf{1}, \mathbf{2}, \mathbf{1}, \mathbf{1}, \mathbf{1}, \mathbf{1})$ & $(\mathbf{1}, \mathbf{3}, \mathbf{0}, \mathbf{1})$ & $(2,3,2,2)$ & 1 & $\mp 1$ & $2 k$ & $2 k$ & $\mp 2$ & $\mp 2^{15}$ & $\mp 1$ & 33 \\
\hline
\end{tabular}


Table 5. (continued)

\begin{tabular}{|c|c|c|c|c|c|c|c|c|c|c|c|}
\hline & $\phi$ & $\psi$ & $\omega$ & & $z_{1}$ & $z_{2}$ & $z_{3}$ & $z_{4}$ & $z_{5}$ & $z_{6}$ & \# of free bits \\
\hline 157 & $(\mathbf{1}, \mathbf{0}, \mathbf{1}, \mathbf{1}, \mathbf{1}, \mathbf{0})$ & $(\mathbf{1}, \mathbf{0}, \mathbf{0}, \mathbf{3})$ & $(\mathbf{2}, \mathbf{3}, \mathbf{0}, \mathbf{1})$ & 1 & $\mp 1$ & - & $2 k$ & $\mp 2^{15}$ & $\mp 2^{15}$ & - & 50 \\
\hline 158 & $(\mathbf{1}, \mathbf{1}, \mathbf{1}, \mathbf{1}, \mathbf{1}, \mathbf{1})$ & $(\mathbf{1}, \mathbf{1}, \mathbf{0}, \mathbf{3})$ & $(2,3,0,0)$ & 1 & $\mp 1$ & - & $2 k+1$ & $\mp 2^{15}$ & $\mp 2^{15}$ & $\mp 1$ & 35 \\
\hline 159 & $(\mathbf{0}, \mathbf{1}, \mathbf{2}, \mathbf{0}, \mathbf{0}, \mathbf{1})$ & $(0,1,2,0)$ & $(\mathbf{0}, \mathbf{2}, \mathbf{0}, \mathbf{1})$ & 0 & - & - & $2 k$ & - & - & $\mp 2$ & 79 \\
\hline 160 & $(\mathbf{1}, \mathbf{0}, \mathbf{2}, \mathbf{0}, \mathbf{1}, \mathbf{1})$ & $(3,0,2,0)$ & $(\mathbf{0}, \mathbf{3}, \mathbf{1}, \mathbf{1})$ & 0 & $\mp 2^{15}$ & - & $2 k$ & - & $\mp 1$ & $\mp 2$ & 49 \\
\hline 161 & $(\mathbf{1}, \mathbf{0}, \mathbf{3}, \mathbf{0}, \mathbf{1}, \mathbf{1})$ & $(\mathbf{1}, \mathbf{0}, \mathbf{3}, \mathbf{0})$ & $(\mathbf{2}, \mathbf{3}, \mathbf{1}, \mathbf{1})$ & 1 & $\mp 2$ & - & $2 k+1$ & - & $\mp 1$ & $\mp 1$ & 49 \\
\hline 162 & $(1,1,1,0,1,0)$ & $(\mathbf{1}, \mathbf{1}, \mathbf{0}, \mathbf{0})$ & $(2,3,1,0)$ & 0 & $\mp 1$ & - & $2 k$ & - & $\mp 2^{15}$ & - & 65 \\
\hline 163 & $(1,2,0,1,1,1)$ & $(\mathbf{1}, \mathbf{3}, \mathbf{0}, \mathbf{1})$ & $(0,1,2,2)$ & 1 & $\mp 1$ & $2 k$ & - & $\mp 2$ & $\mp 1$ & $\mp 1$ & 34 \\
\hline 164 & $(\mathbf{1}, \mathbf{3}, \mathbf{1}, \mathbf{1}, \mathbf{0}, \mathbf{1})$ & $(\mathbf{1}, \mathbf{3}, \mathbf{1}, \mathbf{1})$ & $(\mathbf{1}, \mathbf{1}, \mathbf{3}, \mathbf{2})$ & 1 & $\mp 1$ & $2 k+1$ & - & $\mp 2$ & - & $\mp 1$ & 49 \\
\hline 165 & $(\mathbf{1}, \mathbf{3}, \mathbf{3}, \mathbf{1}, \mathbf{0}, \mathbf{1})$ & $(\mathbf{3}, \mathbf{2}, \mathbf{3}, \mathbf{1})$ & $(\mathbf{1}, \mathbf{3}, \mathbf{3}, \mathbf{2})$ & 1 & $\mp 2^{15}$ & $2 k$ & $2 k$ & $\mp 2$ & - & $\mp 2$ & 47 \\
\hline 166 & $(\mathbf{1}, \mathbf{3}, \mathbf{3}, \mathbf{1}, \mathbf{1}, \mathbf{0})$ & $(1,2,2,1)$ & $(2,3,2,3)$ & 1 & $\mp 2$ & $2 k$ & $2 k$ & $\mp 2$ & $\mp 1$ & - & 47 \\
\hline 167 & $(\mathbf{1}, \mathbf{0}, \mathbf{3}, \mathbf{1}, \mathbf{0}, \mathbf{1})$ & $(1,0,2,1)$ & $(\mathbf{1}, \mathbf{3}, \mathbf{1}, \mathbf{0})$ & 0 & $\mp 1$ & - & $2 k+1$ & $\mp 1$ & - & $\mp 2$ & 49 \\
\hline 168 & $(\mathbf{1}, \mathbf{0}, \mathbf{2}, \mathbf{0}, \mathbf{1}, \mathbf{1})$ & $(\mathbf{1}, \mathbf{0}, \mathbf{3}, \mathbf{0})$ & $(\mathbf{0}, \mathbf{3}, \mathbf{1}, \mathbf{1})$ & 0 & $\mp 1$ & - & $2 k+1$ & - & $\mp 1$ & $\mp 2$ & 49 \\
\hline 169 & $(1,0,3,1,1,0)$ & $(\mathbf{1}, \mathbf{0}, \mathbf{3}, \mathbf{1})$ & $(\mathbf{2}, \mathbf{3}, \mathbf{0}, \mathbf{1})$ & 1 & $\mp 2$ & - & $2 k+1$ & $\mp 1$ & $\mp 1$ & - & 49 \\
\hline 170 & $(\mathbf{1}, \mathbf{1}, \mathbf{2}, \mathbf{1}, \mathbf{1}, \mathbf{1})$ & $(\mathbf{3}, \mathbf{1}, \mathbf{3}, \mathbf{3})$ & $(\mathbf{0}, \mathbf{3}, \mathbf{0}, \mathbf{0})$ & 1 & $\mp 2^{15}$ & - & $2 k+1$ & $\mp 2^{15}$ & $\mp 1$ & $\mp 2$ & 34 \\
\hline 171 & $(\mathbf{1}, \mathbf{3}, \mathbf{2}, \mathbf{1}, \mathbf{0}, \mathbf{1})$ & $(\mathbf{1}, \mathbf{3}, \mathbf{2}, \mathbf{1})$ & $(\mathbf{3}, \mathbf{3}, \mathbf{3}, \mathbf{2})$ & 1 & $\mp 2$ & $2 k+1$ & $2 k+1$ & $\mp 2$ & - & $\mp 1$ & 47 \\
\hline 172 & $(\mathbf{1}, \mathbf{3}, \mathbf{3}, \mathbf{1}, \mathbf{0}, \mathbf{1})$ & $(3,3,2,1)$ & $(1,3,3,2)$ & 0 & $\mp 2^{15}$ & $2 k+1$ & $2 k+1$ & $\mp 2$ & - & $\mp 2$ & 47 \\
\hline 173 & $(\mathbf{1}, \mathbf{3}, \mathbf{1}, \mathbf{1}, \mathbf{1}, \mathbf{0})$ & $(\mathbf{3}, \mathbf{2}, \mathbf{0}, \mathbf{1})$ & $(2,3,2,3)$ & 1 & $\mp 2^{15}$ & $2 k$ & $2 k+1$ & $\mp 2$ & $\mp 2^{15}$ & - & 48 \\
\hline 174 & $(1,1,2,1,0,0)$ & $(1,1,2,3)$ & $(\mathbf{3}, \mathbf{3}, \mathbf{1}, \mathbf{1})$ & 0 & $\mp 2$ & - & $2 k+1$ & $\mp 2^{15}$ & - & - & 64 \\
\hline 175 & $(\mathbf{0}, \mathbf{0}, \mathbf{2}, \mathbf{0}, \mathbf{1}, \mathbf{1})$ & $(\mathbf{0}, \mathbf{0}, \mathbf{2}, \mathbf{0})$ & $(\mathbf{3}, \mathbf{0}, \mathbf{1}, \mathbf{1})$ & 0 & - & - & $2 k+1$ & - & $\mp 2^{15}$ & $\mp 2$ & 64 \\
\hline 176 & $(\mathbf{0}, \mathbf{3}, \mathbf{0}, \mathbf{1}, \mathbf{0}, \mathbf{1})$ & $(\mathbf{0}, \mathbf{3}, \mathbf{0}, \mathbf{1})$ & $(\mathbf{0}, \mathbf{0}, \mathbf{3}, \mathbf{2})$ & 0 & - & $2 k+1$ & - & $\mp 2$ & - & $\mp 1$ & 64 \\
\hline 177 & $(\mathbf{0}, \mathbf{3}, \mathbf{0}, \mathbf{1}, \mathbf{1}, \mathbf{0})$ & $(\mathbf{0}, \mathbf{3}, \mathbf{1}, \mathbf{1})$ & $(3,2,2,3)$ & 0 & - & $2 k+1$ & $2 k$ & $\mp 2$ & $\mp 2^{15}$ & - & 63 \\
\hline 178 & $(\mathbf{0}, \mathbf{0}, \mathbf{3}, \mathbf{0}, \mathbf{1}, \mathbf{1})$ & $(\mathbf{0}, \mathbf{0}, \mathbf{3}, \mathbf{0})$ & $(\mathbf{1}, \mathbf{2}, \mathbf{1}, \mathbf{1})$ & 1 & - & - & $2 k$ & - & $\mp 1$ & $\mp 2$ & 64 \\
\hline 179 & $(\mathbf{1}, \mathbf{1}, \mathbf{3}, \mathbf{0}, \mathbf{0}, \mathbf{1})$ & $(\mathbf{3}, \mathbf{1}, \mathbf{3}, \mathbf{0})$ & $(\mathbf{1}, \mathbf{3}, \mathbf{0}, \mathbf{1})$ & 1 & $\mp 2^{15}$ & - & $2 k$ & - & - & $\mp 2$ & 64 \\
\hline 180 & $(\mathbf{0}, \mathbf{3}, \mathbf{2}, \mathbf{1}, \mathbf{0}, \mathbf{1})$ & $(\mathbf{0}, \mathbf{2}, \mathbf{3}, \mathbf{1})$ & $(\mathbf{0}, \mathbf{2}, \mathbf{3}, \mathbf{2})$ & 0 & - & $2 k$ & $2 k+1$ & $\mp 2$ & - & $\mp 2$ & 62 \\
\hline 181 & $(\mathbf{1}, \mathbf{1}, \mathbf{3}, \mathbf{1}, \mathbf{1}, \mathbf{1})$ & $(\mathbf{1}, \mathbf{1}, 2,3)$ & $(2,3,0,0)$ & 1 & $\mp 2$ & - & $2 k$ & $\mp 2^{15}$ & $\mp 1$ & $\mp 1$ & 34 \\
\hline 182 & $(\mathbf{1}, \mathbf{2}, \mathbf{0}, \mathbf{1}, \mathbf{1}, \mathbf{1})$ & $(\mathbf{1}, \mathbf{2}, \mathbf{0}, \mathbf{1})$ & $(\mathbf{0}, \mathbf{1}, \mathbf{2}, \mathbf{2})$ & 0 & $\mp 1$ & $2 k+1$ & - & $\mp 2$ & $\mp 1$ & $\mp 1$ & 34 \\
\hline 183 & $(\mathbf{1}, \mathbf{1}, \mathbf{3}, \mathbf{1}, \mathbf{1}, \mathbf{1})$ & $(3,1,2,1)$ & $(\mathbf{2}, \mathbf{1}, \mathbf{0}, \mathbf{0})$ & 1 & $\mp 2^{15}$ & - & $2 k$ & $\mp 1$ & $\mp 2^{15}$ & $\mp 2$ & 34 \\
\hline 184 & $(1,3,2,1,1,0)$ & $(\mathbf{1}, \mathbf{2}, \mathbf{3}, \mathbf{1})$ & $(\mathbf{0}, \mathbf{1}, \mathbf{2}, \mathbf{3})$ & 1 & $\mp 2$ & $2 k$ & $2 k+1$ & $\mp 2$ & $\mp 2^{15}$ & - & 47 \\
\hline 185 & $(1,0,2,0,0,0)$ & $(1,0,2,0)$ & $(\mathbf{3}, \mathbf{3}, \mathbf{0}, \mathbf{0})$ & 1 & $\mp 2$ & - & $2 k+1$ & - & - & - & 79 \\
\hline 186 & $(\mathbf{1}, \mathbf{0}, \mathbf{3}, \mathbf{0}, \mathbf{1}, \mathbf{1})$ & $(\mathbf{1}, \mathbf{0}, \mathbf{3}, \mathbf{0})$ & $(\mathbf{2}, \mathbf{1}, \mathbf{1}, \mathbf{1})$ & 1 & $\mp 1$ & - & $2 k+1$ & - & $\mp 2^{15}$ & $\mp 2$ & 49 \\
\hline 187 & $(\mathbf{0}, \mathbf{2}, \mathbf{2}, \mathbf{1}, \mathbf{1}, \mathbf{1})$ & $(\mathbf{0}, \mathbf{2}, \mathbf{3}, \mathbf{1})$ & $(3,0,2,2)$ & 0 & - & $2 k+1$ & $2 k$ & $\mp 2$ & $\mp 2^{15}$ & $\mp 2$ & 47 \\
\hline 188 & $(1,2,2,1,1,1)$ & $(\mathbf{1}, \mathbf{3}, \mathbf{2}, \mathbf{1})$ & $(\mathbf{0}, 3,2,2)$ & 0 & $\mp 1$ & $2 k$ & $2 k$ & $\mp 2$ & $\mp 1$ & $\mp 2$ & 32 \\
\hline 189 & $(\mathbf{1}, \mathbf{3}, \mathbf{3}, \mathbf{1}, \mathbf{0}, \mathbf{1})$ & $(\mathbf{1}, \mathbf{3}, \mathbf{3}, \mathbf{1})$ & $(\mathbf{1}, \mathbf{3}, \mathbf{3}, \mathbf{2})$ & 0 & $\mp 1$ & $2 k+1$ & $2 k$ & $\mp 2$ & - & $\mp 2$ & 47 \\
\hline 190 & $(\mathbf{1}, \mathbf{0}, \mathbf{2}, \mathbf{0}, \mathbf{1}, \mathbf{1})$ & $(1,0,2,0)$ & $(\mathbf{0}, \mathbf{1}, \mathbf{1}, \mathbf{1})$ & 0 & $\mp 2$ & - & $2 k$ & - & $\mp 2^{15}$ & $\mp 1$ & 49 \\
\hline 191 & $(\mathbf{1}, \mathbf{3}, \mathbf{1}, \mathbf{1}, \mathbf{0}, \mathbf{1})$ & $(\mathbf{3}, \mathbf{3}, \mathbf{1}, \mathbf{1})$ & $(\mathbf{1}, \mathbf{1}, \mathbf{3}, \mathbf{2})$ & 1 & $\mp 2^{15}$ & $2 k+1$ & - & $\mp 2$ & - & $\mp 1$ & 49 \\
\hline 192 & $(1,0,2,1,0,1)$ & $(1,0,2,1)$ & $(\mathbf{3}, \mathbf{3}, \mathbf{1}, \mathbf{0})$ & 1 & $\mp 2$ & - & $2 k+1$ & $\mp 1$ & - & $\mp 1$ & 49 \\
\hline 193 & $(\mathbf{1}, \mathbf{0}, \mathbf{3}, \mathbf{1}, \mathbf{0}, \mathbf{1})$ & $(3,0,2,1)$ & $(\mathbf{1}, \mathbf{3}, \mathbf{1}, \mathbf{0})$ & 0 & $\mp 2^{15}$ & - & $2 k+1$ & $\mp 1$ & - & $\mp 2$ & 49 \\
\hline 194 & $(\mathbf{1}, \mathbf{0}, \mathbf{2}, \mathbf{0}, \mathbf{1}, \mathbf{1})$ & $(\mathbf{3}, \mathbf{0}, \mathbf{3}, \mathbf{0})$ & $(\mathbf{0}, \mathbf{3}, \mathbf{1}, \mathbf{1})$ & 0 & $\mp 2^{15}$ & - & $2 k+1$ & - & $\mp 1$ & $\mp 2$ & 49 \\
\hline
\end{tabular}


Table 5. (continued)

\begin{tabular}{|c|c|c|c|c|c|c|c|c|c|c|c|}
\hline & $\phi$ & $\psi$ & $\omega$ & $\lambda$ & $z_{1}$ & $z_{2}$ & $z_{3}$ & $z_{4}$ & $z_{5}$ & $z_{6}$ & \# of free bits \\
\hline 195 & $(\mathbf{0}, \mathbf{2}, \mathbf{2}, \mathbf{1}, \mathbf{1}, \mathbf{1})$ & $(\mathbf{0}, \mathbf{3}, \mathbf{3}, \mathbf{1})$ & $(\mathbf{3}, \mathbf{0}, \mathbf{2}, \mathbf{2})$ & 1 . & - & $2 k$ & $2 k$ & $\mp 2$ & $\mp 2^{15}$ & $\mp 2$ & 47 \\
\hline 196 & $(\mathbf{1}, \mathbf{1}, \mathbf{2}, \mathbf{1}, \mathbf{1}, \mathbf{1})$ & $(\mathbf{1}, \mathbf{1}, \mathbf{3}, \mathbf{3})$ & $(\mathbf{0}, \mathbf{1}, \mathbf{0}, \mathbf{0})$ & 1 & $\mp 2$ & - & $2 k+1$ & $\mp 2^{15}$ & $\mp 2^{15}$ & $\mp 1$ & 34 \\
\hline 197 & $(\mathbf{1}, \mathbf{3}, \mathbf{2}, \mathbf{1}, \mathbf{1}, \mathbf{0})$ & $(\mathbf{1}, \mathbf{3}, \mathbf{2}, \mathbf{1})$ & $(\mathbf{0}, \mathbf{1}, \mathbf{2}, \mathbf{3})$ & 0 & $\mp 2$ & $2 k+1$ & $2 k$ & $\mp 2$ & $\mp 2^{15}$ & - & 47 \\
\hline 198 & $(\mathbf{1}, \mathbf{2}, \mathbf{0}, \mathbf{1}, \mathbf{1}, \mathbf{1})$ & $(\mathbf{1}, \mathbf{2}, \mathbf{0}, \mathbf{1})$ & $(\mathbf{0}, \mathbf{3}, \mathbf{2}, \mathbf{2})$ & 0 & $\mp 2$ & $2 k+1$ & - & $\mp 2$ & $\mp 2^{15}$ & $\mp 2$ & 32 \\
\hline 199 & $(\mathbf{0}, \mathbf{2}, \mathbf{3}, \mathbf{1}, \mathbf{1}, \mathbf{1})$ & $(0,2,2,1)$ & $(1,2,2,2)$ & 0 & - & $2 k+1$ & $2 k+1$ & $\mp 2$ & $\mp 1$ & $\mp 2$ & 47 \\
\hline 200 & $(\mathbf{1}, \mathbf{1}, \mathbf{3}, \mathbf{1}, \mathbf{1}, \mathbf{1})$ & $(\mathbf{1}, \mathbf{1}, \mathbf{2}, \mathbf{3})$ & $(2, \mathbf{1}, \mathbf{0}, \mathbf{0})$ & 1 & $\mp 1$ & - & $2 k$ & $\mp 2^{15}$ & $\mp 2^{15}$ & $\mp 2$ & 34 \\
\hline 201 & $(\mathbf{1}, \mathbf{0}, \mathbf{3}, \mathbf{0}, \mathbf{1}, \mathbf{1})$ & $(\mathbf{1}, \mathbf{0}, \mathbf{2}, \mathbf{0})$ & $(\mathbf{2}, \mathbf{3}, \mathbf{1}, \mathbf{1})$ & 0 & $\mp 2$ & - & $2 k$ & - & $\mp 1$ & $\mp 1$ & 49 \\
\hline 202 & $(\mathbf{0}, \mathbf{3}, \mathbf{2}, \mathbf{1}, \mathbf{0}, \mathbf{1})$ & $(\mathbf{0}, \mathbf{3}, \mathbf{2}, \mathbf{1})$ & $(\mathbf{0}, \mathbf{2}, \mathbf{3}, \mathbf{2})$ & 1 . & - & $2 k+1$ & $2 k$ & $\mp 2$ & - & $\mp 2$ & 62 \\
\hline 203 & $(\mathbf{1}, \mathbf{2}, \mathbf{0}, \mathbf{1}, \mathbf{1}, \mathbf{1})$ & $(3, \mathbf{2}, \mathbf{0}, \mathbf{1})$ & $(0,1,2,2)$ & 0 & $\mp 2^{15}$ & $2 k+1$ & - & $\mp 2$ & $\mp 1$ & $\mp 1$ & 34 \\
\hline 204 & $(\mathbf{1}, \mathbf{2}, \mathbf{0}, \mathbf{1}, \mathbf{1}, \mathbf{1})$ & $(\mathbf{1}, \mathbf{3}, \mathbf{0}, \mathbf{1})$ & $(\mathbf{0}, \mathbf{3}, \mathbf{2}, \mathbf{2})$ & 1 & $\mp 2$ & $2 k$ & - & $\mp 2$ & $\mp 2^{15}$ & $\mp 2$ & 32 \\
\hline 205 & $(\mathbf{0}, \mathbf{2}, \mathbf{3}, \mathbf{1}, \mathbf{1}, \mathbf{1})$ & $(\mathbf{0}, \mathbf{3}, \mathbf{2}, \mathbf{1})$ & $(1,2,2,2)$ & 1 & - & $2 k$ & $2 k+1$ & $\mp 2$ & $\mp 1$ & $\mp 2$ & 47 \\
\hline 206 & $(\mathbf{1}, \mathbf{0}, \mathbf{3}, \mathbf{1}, \mathbf{0}, \mathbf{1})$ & $(\mathbf{1}, \mathbf{0}, \mathbf{3}, \mathbf{1})$ & $(\mathbf{1}, \mathbf{3}, \mathbf{1}, \mathbf{0})$ & 0 & $\mp 1$ & - & $2 k$ & $\mp 1$ & - & $\mp 2$ & 49 \\
\hline 207 & $(\mathbf{1}, \mathbf{0}, \mathbf{3}, \mathbf{0}, \mathbf{1}, \mathbf{1})$ & $(\mathbf{3}, \mathbf{0}, \mathbf{3}, \mathbf{0})$ & $(\mathbf{2}, \mathbf{1}, \mathbf{1}, \mathbf{1})$ & 1 & $\mp 2^{15}$ & - & $2 k+1$ & - & $\mp 2^{15}$ & $\mp 2$ & 49 \\
\hline 208 & $(\mathbf{1}, \mathbf{2}, \mathbf{2}, \mathbf{1}, \mathbf{1}, \mathbf{1})$ & $(1,2,2,1)$ & $(\mathbf{0}, \mathbf{3}, \mathbf{2}, \mathbf{2})$ & 1 & $\mp 1$ & $2 k+1$ & $2 k$ & $\mp 2$ & $\mp 1$ & $\mp 2$ & 32 \\
\hline 209 & $(\mathbf{1}, \mathbf{2}, \mathbf{3}, \mathbf{1}, \mathbf{1}, \mathbf{1})$ & $(\mathbf{1}, \mathbf{3}, \mathbf{3}, \mathbf{1})$ & $(2,3,2,2)$ & 1 & $\mp 2$ & $2 k$ & $2 k+1$ & $\mp 2$ & $\mp 1$ & $\mp 1$ & 32 \\
\hline 210 & $(\mathbf{1}, \mathbf{2}, \mathbf{2}, \mathbf{1}, \mathbf{1}, \mathbf{1})$ & $(3, \mathbf{3}, \mathbf{2}, \mathbf{1})$ & $(0,3,2,2)$ & 0 & $\mp 2^{15}$ & $2 k$ & $2 k$ & $\mp 2$ & $\mp 1$ & $\mp 2$ & 32 \\
\hline 211 & $(\mathbf{1}, \mathbf{3}, \mathbf{3}, \mathbf{1}, \mathbf{1}, \mathbf{0})$ & $(\mathbf{1}, \mathbf{3}, \mathbf{2}, \mathbf{1})$ & $(2,3,2,3)$ & 0 & $\mp 2$ & $2 k+1$ & $2 k$ & $\mp 2$ & $\mp 1$ & - & 47 \\
\hline 212 & $(\mathbf{1}, \mathbf{0}, \mathbf{3}, \mathbf{1}, \mathbf{0}, \mathbf{1})$ & $(1,0,2,3)$ & $(\mathbf{1}, \mathbf{3}, \mathbf{1}, \mathbf{0})$ & 0 & $\mp 1$ & - & $2 k+1$ & $\mp 2^{15}$ & - & $\mp 2$ & 49 \\
\hline 213 & $(\mathbf{1}, \mathbf{3}, \mathbf{3}, \mathbf{1}, \mathbf{0}, \mathbf{1})$ & $(\mathbf{3}, \mathbf{3}, \mathbf{3}, \mathbf{1})$ & $(\mathbf{1}, \mathbf{3}, \mathbf{3}, \mathbf{2})$ & 0 & $\mp 2^{15}$ & $2 k+1$ & $2 k$ & $\mp 2$ & - & $\mp 2$ & 47 \\
\hline 214 & $(\mathbf{1}, \mathbf{2}, \mathbf{0}, \mathbf{1}, \mathbf{1}, \mathbf{1})$ & $(\mathbf{3}, \mathbf{3}, \mathbf{0}, \mathbf{1})$ & $(\mathbf{0}, \mathbf{1}, \mathbf{2}, \mathbf{2})$ & 1 & $\mp 2^{15}$ & $2 k$ & - & $\mp 2$ & $\mp 1$ & $\mp 1$ & 34 \\
\hline 215 & $(1,2,2,1,0,0)$ & $(\mathbf{1}, \mathbf{2}, 3, \mathbf{1})$ & $(\mathbf{3}, \mathbf{3}, \mathbf{3}, \mathbf{3})$ & 1 & $\mp 2$ & $2 k+1$ & $2 k$ & $\mp 2$ & - & - & 62 \\
\hline 216 & $(\mathbf{0}, \mathbf{1}, \mathbf{2}, \mathbf{0}, \mathbf{0}, \mathbf{1})$ & $(\mathbf{0}, \mathbf{1}, \mathbf{3}, \mathbf{0})$ & $(\mathbf{0}, \mathbf{2}, \mathbf{0}, \mathbf{1})$ & 0 & - & - & $2 k+1$ & - & - & $\mp 2$ & 79 \\
\hline 217 & $(1,2,2,1,1,1)$ & $(\mathbf{1}, \mathbf{3}, \mathbf{3}, \mathbf{1})$ & $(\mathbf{0}, \mathbf{3}, \mathbf{2}, \mathbf{2})$ & 0 & $\mp 1$ & $2 k$ & $2 k+1$ & $\mp 2$ & $\mp 1$ & $\mp 2$ & 32 \\
\hline 218 & $(0,2,1,1,1,1)$ & $(\mathbf{0}, \mathbf{3}, \mathbf{1}, \mathbf{1})$ & $(\mathbf{1}, \mathbf{0}, \mathbf{2}, \mathbf{2})$ & 0 & - & $2 k$ & - & $\mp 2$ & $\mp 1$ & $\mp 1$ & 49 \\
\hline 219 & $(\mathbf{1}, \mathbf{1}, \mathbf{3}, \mathbf{0}, \mathbf{1}, \mathbf{0})$ & $(1, \mathbf{1}, \mathbf{3}, \mathbf{0})$ & $(2,3, \mathbf{1}, \mathbf{0})$ & 0 & $\mp 2$ & - & $2 k+1$ & - & $\mp 1$ & - & 64 \\
\hline 220 & $(\mathbf{0}, \mathbf{2}, \mathbf{1}, \mathbf{1}, \mathbf{1}, \mathbf{1})$ & $(\mathbf{0}, \mathbf{2}, \mathbf{1}, \mathbf{1})$ & $(\mathbf{1}, \mathbf{0}, \mathbf{2}, \mathbf{2})$ & 1 & - & $2 k+1$ & - & $\mp 2$ & $\mp 1$ & $\mp 1$ & 49 \\
\hline 221 & $(\mathbf{0}, \mathbf{0}, \mathbf{2}, \mathbf{1}, \mathbf{0}, \mathbf{1})$ & $(\mathbf{0}, \mathbf{0}, \mathbf{2}, \mathbf{1})$ & $(\mathbf{0}, \mathbf{2}, \mathbf{1}, \mathbf{0})$ & 1 & - & - & $2 k$ & $\mp 1$ & - & $\mp 2$ & 64 \\
\hline 222 & $(\mathbf{1}, \mathbf{0}, \mathbf{3}, \mathbf{0}, \mathbf{1}, \mathbf{1})$ & $(\mathbf{1}, \mathbf{0}, \mathbf{2}, \mathbf{0})$ & $(\mathbf{2}, \mathbf{1}, \mathbf{1}, \mathbf{1})$ & 0 & $\mp 1$ & - & $2 k$ & - & $\mp 2^{15}$ & $\mp 2$ & 49 \\
\hline 223 & $(\mathbf{1}, \mathbf{1}, \mathbf{3}, \mathbf{1}, \mathbf{1}, \mathbf{1})$ & $(\mathbf{3}, \mathbf{1}, \mathbf{2}, \mathbf{3})$ & $(\mathbf{2}, \mathbf{1}, \mathbf{0}, \mathbf{0})$ & 1 & $\mp 2^{15}$ & - & $2 k$ & $\mp 2^{15}$ & $\mp 2^{15}$ & $\mp 2$ & 34 \\
\hline 224 & $(\mathbf{1}, \mathbf{3}, \mathbf{2}, \mathbf{1}, \mathbf{1}, \mathbf{0})$ & $(\mathbf{1}, \mathbf{3}, \mathbf{3}, \mathbf{1})$ & $(\mathbf{0}, \mathbf{1}, \mathbf{2}, \mathbf{3})$ & 0 & $\mp 2$ & $2 k+1$ & $2 k+1$ & $\mp 2$ & $\mp 2^{15}$ & - & 47 \\
\hline 225 & $(1,2,3,1,1,1)$ & $(\mathbf{1}, \mathbf{2}, \mathbf{3}, \mathbf{1})$ & $(\mathbf{2}, \mathbf{3}, \mathbf{2}, \mathbf{2})$ & 0 & $\mp 2$ & $2 k+1$ & $2 k+1$ & $\mp 2$ & $\mp 1$ & $\mp 1$ & 32 \\
\hline 226 & $(\mathbf{0}, \mathbf{1}, \mathbf{3}, \mathbf{1}, \mathbf{1}, \mathbf{1})$ & $(\mathbf{0}, \mathbf{1}, \mathbf{2}, \mathbf{1})$ & $(\mathbf{1}, \mathbf{2}, \mathbf{0}, \mathbf{0})$ & 0 & - & - & $2 k+1$ & $\mp 1$ & $\mp 1$ & $\mp 2$ & 49 \\
\hline 227 & $(\mathbf{1}, \mathbf{2}, \mathbf{3}, \mathbf{1}, \mathbf{1}, \mathbf{1})$ & $(\mathbf{1}, \mathbf{3}, \mathbf{3}, \mathbf{1})$ & $(2,1,2,2)$ & 1 & $\mp 1$ & $2 k$ & $2 k+1$ & $\mp 2$ & $\mp 2^{15}$ & $\mp 2$ & 32 \\
\hline 228 & $(\mathbf{1}, \mathbf{0}, \mathbf{3}, \mathbf{1}, \mathbf{0}, \mathbf{1})$ & $(\mathbf{3}, \mathbf{0}, \mathbf{3}, \mathbf{1})$ & $(\mathbf{1}, \mathbf{3}, \mathbf{1}, \mathbf{0})$ & 0 & $\mp 2^{15}$ & - & $2 k$ & $\mp 1$ & - & $\mp 2$ & 49 \\
\hline 229 & $(\mathbf{1}, \mathbf{0}, \mathbf{3}, \mathbf{1}, \mathbf{1}, \mathbf{0})$ & $(\mathbf{1}, \mathbf{0}, \mathbf{2}, \mathbf{1})$ & $(\mathbf{2}, \mathbf{3}, \mathbf{0}, \mathbf{1})$ & 0 & $\mp 2$ & - & $2 k$ & $\mp 1$ & $\mp 1$ & - & 49 \\
\hline 230 & $(\mathbf{1}, \mathbf{0}, \mathbf{2}, \mathbf{1}, \mathbf{0}, \mathbf{1})$ & $(\mathbf{1}, \mathbf{0}, \mathbf{2}, \mathbf{3})$ & $(\mathbf{3}, \mathbf{3}, \mathbf{1}, \mathbf{0})$ & 1 & $\mp 2$ & - & $2 k+1$ & $\mp 2^{15}$ & - & $\mp 1$ & 49 \\
\hline 231 & $(\mathbf{1}, \mathbf{0}, \mathbf{3}, \mathbf{1}, \mathbf{0}, \mathbf{1})$ & $(\mathbf{3}, \mathbf{0}, \mathbf{2}, \mathbf{3})$ & $(\mathbf{1}, \mathbf{3}, \mathbf{1}, \mathbf{0})$ & 0 & $\mp 2^{15}$ & - & $2 k+1$ & $\mp 2^{15}$ & - & $\mp 2$ & 49 \\
\hline 232 & $(\mathbf{1}, \mathbf{2}, \mathbf{2}, \mathbf{1}, \mathbf{1}, \mathbf{1})$ & $(3,2,2,1)$ & $(0,3,2,2)$ & 1 & $\mp 2^{15}$ & $2 k+1$ & $2 k$ & $\mp 2$ & $\mp 1$ & $\mp 2$ & 32 \\
\hline 233 & $(\mathbf{1}, \mathbf{0}, \mathbf{3}, \mathbf{1}, \mathbf{1}, \mathbf{0})$ & $(\mathbf{1}, \mathbf{0}, \mathbf{3}, \mathbf{3})$ & $(\mathbf{2}, \mathbf{3}, \mathbf{0}, \mathbf{1})$ & 1 & $\mp 2$ & - & $2 k+1$ & $\mp 2^{15}$ & $\mp 1$ & - & 49 \\
\hline
\end{tabular}


Table 5. (continued)

\begin{tabular}{|c|c|c|c|c|c|c|c|c|c|c|c|}
\hline & $\phi$ & $\psi$ & $\omega$ & & $z_{1}$ & $z_{2}$ & $z_{3}$ & $z_{4}$ & $z_{5}$ & $z_{6}$ & \# of free bits \\
\hline 234 & $(\mathbf{1}, 2,2,1,1,1)$ & $(\mathbf{1}, \mathbf{2}, \mathbf{3}, \mathbf{1})$ & $(\mathbf{0}, 3,2,2)$ & 1 & $\mp 1$ & $2 k+1$ & $2 k+1$ & $\mp 2$ & $\mp 1$ & $\mp 2$ & 32 \\
\hline 235 & $(\mathbf{1}, \mathbf{1}, \mathbf{2}, \mathbf{1}, \mathbf{1}, \mathbf{1})$ & $(\mathbf{1}, \mathbf{1}, \mathbf{2}, \mathbf{1})$ & $(\mathbf{0}, \mathbf{3}, \mathbf{0}, \mathbf{0})$ & 1 & $\mp 1$ & - & $2 k$ & $\mp 1$ & $\mp 1$ & $\mp 2$ & 34 \\
\hline 236 & $(\mathbf{1}, \mathbf{3}, \mathbf{0}, \mathbf{1}, \mathbf{1}, \mathbf{0})$ & $(\mathbf{1}, \mathbf{2}, \mathbf{0}, \mathbf{1})$ & $(0, \mathbf{1}, \mathbf{2}, \mathbf{3})$ & 0 & $\mp 1$ & $2 k$ & - & $\mp 2$ & $\mp 1$ & - & 49 \\
\hline 237 & $(\mathbf{0}, \mathbf{2}, \mathbf{3}, \mathbf{1}, \mathbf{1}, \mathbf{1})$ & $(\mathbf{0}, \mathbf{2}, \mathbf{3}, \mathbf{1})$ & $(1,2,2,2)$ & 0 & - & $2 k+1$ & $2 k$ & $\mp 2$ & $\mp 1$ & $\mp 2$ & 47 \\
\hline 238 & $(\mathbf{1}, \mathbf{0}, \mathbf{2}, \mathbf{0}, \mathbf{1}, \mathbf{1})$ & $(\mathbf{1}, \mathbf{0}, \mathbf{3}, \mathbf{0})$ & $(\mathbf{0}, \mathbf{1}, \mathbf{1}, \mathbf{1})$ & 0 & $\mp 2$ & - & $2 k+1$ & - & $\mp 2^{15}$ & $\mp 1$ & 49 \\
\hline 239 & $(\mathbf{0}, \mathbf{2}, \mathbf{2}, \mathbf{1}, \mathbf{1}, \mathbf{1})$ & $(\mathbf{0}, \mathbf{2}, \mathbf{2}, \mathbf{1})$ & $(3,0,2,2)$ & 1 & - & $2 k+1$ & $2 k+1$ & $\mp 2$ & $\mp 2^{15}$ & $\mp 2$ & 47 \\
\hline 240 & $(\mathbf{0}, \mathbf{2}, \mathbf{2}, \mathbf{1}, \mathbf{1}, \mathbf{1})$ & $(\mathbf{0}, \mathbf{3}, \mathbf{2}, \mathbf{1})$ & $(3,0,2,2)$ & 0 & - & $2 k$ & $2 k+1$ & $\mp 2$ & $\mp 2^{15}$ & $\mp 2$ & 47 \\
\hline 241 & $(\mathbf{0}, \mathbf{2}, \mathbf{3}, \mathbf{1}, \mathbf{1}, \mathbf{1})$ & $(\mathbf{0}, \mathbf{3}, \mathbf{3}, \mathbf{1})$ & $(1,2,2,2)$ & 1 & - & $2 k$ & $2 k$ & $\mp 2$ & $\mp 1$ & $\mp 2$ & 47 \\
\hline 242 & $(\mathbf{1}, \mathbf{0}, \mathbf{2}, \mathbf{1}, \mathbf{1}, \mathbf{0})$ & $(\mathbf{1}, \mathbf{0}, \mathbf{3}, \mathbf{1})$ & $(\mathbf{0}, \mathbf{1}, \mathbf{0}, \mathbf{1})$ & 0 & $\mp 2$ & - & $2 k+1$ & $\mp 1$ & $\mp 2^{15}$ & - & 49 \\
\hline 243 & $(\mathbf{1}, \mathbf{2}, \mathbf{3}, \mathbf{1}, \mathbf{1}, \mathbf{1})$ & $(\mathbf{1}, \mathbf{2}, \mathbf{3}, \mathbf{1})$ & $(2,1,2,2)$ & 0 & $\mp 1$ & $2 k+1$ & $2 k+1$ & $\mp 2$ & $\mp 2^{15}$ & $\mp 2$ & 32 \\
\hline 244 & $(\mathbf{1}, \mathbf{0}, \mathbf{3}, \mathbf{1}, \mathbf{0}, \mathbf{1})$ & $(\mathbf{1}, \mathbf{0}, \mathbf{3}, \mathbf{3})$ & $(\mathbf{1}, \mathbf{3}, \mathbf{1}, \mathbf{0})$ & 0 & $\mp 1$ & - & $2 k$ & $\mp 2^{15}$ & \begin{tabular}{|l|l|}
- \\
\end{tabular} & $\mp 2$ & 49 \\
\hline 245 & $(\mathbf{1}, \mathbf{1}, \mathbf{0}, \mathbf{1}, \mathbf{1}, \mathbf{1})$ & $(\mathbf{1}, \mathbf{1}, \mathbf{0}, \mathbf{3})$ & $(\mathbf{0}, \mathbf{1}, \mathbf{0}, \mathbf{0})$ & 0 & $\mp 1$ & - & - & $\mp 2^{15}$ & $\mp 1$ & $\mp 1$ & 36 \\
\hline 246 & $(\mathbf{0}, \mathbf{3}, \mathbf{2}, \mathbf{1}, \mathbf{0}, \mathbf{1})$ & $(\mathbf{0}, \mathbf{3}, \mathbf{3}, \mathbf{1})$ & $(\mathbf{0}, \mathbf{2}, \mathbf{3}, \mathbf{2})$ & 1 & - & $2 k+1$ & $2 k+1$ & $\mp 2$ & - & $\mp 2$ & 62 \\
\hline 247 & $(\mathbf{1}, \mathbf{1}, \mathbf{3}, \mathbf{1}, \mathbf{1}, \mathbf{1})$ & $(\mathbf{1}, \mathbf{1}, \mathbf{3}, \mathbf{1})$ & $(2, \mathbf{3}, \mathbf{0}, \mathbf{0})$ & 0 & $\mp 2$ & - & $2 k+1$ & $\mp 1$ & $\mp 1$ & $\mp 1$ & 34 \\
\hline 248 & $(\mathbf{1}, \mathbf{3}, \mathbf{2}, \mathbf{1}, \mathbf{0}, \mathbf{1})$ & $(\mathbf{1}, \mathbf{2}, \mathbf{3}, \mathbf{1})$ & $(\mathbf{3}, \mathbf{3}, \mathbf{3}, \mathbf{2})$ & 1 & $\mp 2$ & $2 k$ & $2 k$ & $\mp 2$ & - & $\mp 1$ & 47 \\
\hline 249 & $(\mathbf{1}, \mathbf{2}, \mathbf{2}, \mathbf{1}, \mathbf{1}, \mathbf{1})$ & $(\mathbf{1}, \mathbf{3}, \mathbf{2}, \mathbf{1})$ & $(\mathbf{0}, \mathbf{1}, \mathbf{2}, \mathbf{2})$ & 0 & $\mp 2$ & $2 k$ & $2 k$ & $\mp 2$ & $\mp 2^{15}$ & $\mp 1$ & 32 \\
\hline 250 & $(\mathbf{1}, \mathbf{2}, \mathbf{2}, \mathbf{1}, \mathbf{1}, \mathbf{1})$ & $(\mathbf{1}, \mathbf{2}, \mathbf{2}, \mathbf{1})$ & $(0,1,2,2)$ & 1 & $\mp 2$ & $2 k+1$ & $2 k$ & $\mp 2$ & $\mp 2^{15}$ & $\mp 1$ & 32 \\
\hline 251 & $(\mathbf{1}, \mathbf{2}, \mathbf{1}, \mathbf{1}, \mathbf{0}, \mathbf{0})$ & $(\mathbf{1}, \mathbf{2}, \mathbf{1}, \mathbf{1})$ & $(\mathbf{1}, \mathbf{1}, \mathbf{3}, \mathbf{3})$ & 0 & $\mp 1$ & $2 k+1$ & - & $\mp 2$ & \begin{tabular}{|l|l|}
- & \\
\end{tabular} & - & 64 \\
\hline 252 & $(1,2,2,1,1,1)$ & $(\mathbf{3}, \mathbf{3}, \mathbf{3}, \mathbf{1})$ & $(0,3,2,2)$ & 0 & $\mp 2^{15}$ & $2 k$ & $2 k+1$ & $\mp 2$ & $\mp 1$ & $\mp 2$ & 32 \\
\hline 253 & $(\mathbf{1}, \mathbf{1}, \mathbf{2}, \mathbf{1}, \mathbf{1}, \mathbf{1})$ & $(3, \mathbf{1}, \mathbf{2}, \mathbf{1})$ & $(\mathbf{0}, \mathbf{3}, \mathbf{0}, \mathbf{0})$ & 1 & $\mp 2^{15}$ & - & $2 k$ & $\mp 1$ & $\mp 1$ & $\mp 2$ & 34 \\
\hline 254 & $(1,2,2,1,1,1)$ & $(\mathbf{3}, \mathbf{2}, \mathbf{3}, \mathbf{1})$ & $(0,3,2,2)$ & 1 & $\mp 2^{15}$ & $2 k+1$ & $2 k+1$ & $\mp 2$ & $\mp 1$ & $\mp 2$ & 32 \\
\hline 255 & $(\mathbf{1}, \mathbf{3}, \mathbf{0}, \mathbf{1}, \mathbf{1}, \mathbf{0})$ & $(\mathbf{3}, \mathbf{2}, \mathbf{0}, \mathbf{1})$ & $(\mathbf{0}, \mathbf{1}, \mathbf{2}, \mathbf{3})$ & 0 & $\mp 2^{15}$ & $2 k$ & - & $\mp 2$ & $\mp 1$ & - & 49 \\
\hline
\end{tabular}

Table 6. 50 linear relations with less number of key bits restriction for 8.5-round IDEA cipher. Here each row is associated with one such relation, a linear mask for each round input and one for the last round output, namely ciphertext are provided. Last column shows the number of key bits from the master key that are not restricted, that is, each such bit can be either 0 or 1 . Note that mask $(\mathbf{a}, \mathbf{b}, \mathbf{c}, \mathbf{d})$ is denoted by abcd. When $832-556=276$ key bits are restricted according to Tables 1 and 2 , twenty second row of this table gives a linear relation for 8.5-round IDEA cipher involving plaintext bit $(\mathbf{0}, \mathbf{1}, \mathbf{0}, \mathbf{0}) \star\left(\mathbf{X}_{1}^{0}, \mathbf{X}_{2}^{0}, \mathbf{X}_{3}^{0}, \mathbf{X}_{4}^{0}\right)=\mathbf{1} \cdot \mathbf{X}_{2}^{0}$ and ciphertext bits added $(\mathbf{1}, \mathbf{2}, \mathbf{1}, \mathbf{3}) \star\left(\mathbf{Y}_{1}, \mathbf{Y}_{2}, \mathbf{Y}_{3}, \mathbf{Y}_{4}\right)=\mathbf{1} \cdot \mathbf{Y}_{1} \oplus \mathbf{2} \cdot \mathbf{Y}_{2} \oplus \mathbf{1} \cdot \mathbf{Y}_{3} \oplus \mathbf{3} \cdot \mathbf{Y}_{4}$ (see Sect. 4.2 and Fig. 1 in Appendix A).

\begin{tabular}{|c|c|c|c|c|c|c|c|c|c|c|c|}
\hline \# & $\begin{array}{l}1^{s t} \\
\text { round's } \\
\text { input } \\
\text { mask }\end{array}$ & \begin{tabular}{|l|}
$2^{n d}$ \\
round's \\
input \\
mask
\end{tabular} & $\begin{array}{l}3^{r d} \\
\text { round's } \\
\text { input } \\
\text { mask }\end{array}$ & $\begin{array}{l}4^{t h} \\
\text { round's } \\
\text { input } \\
\text { mask }\end{array}$ & $\begin{array}{l}5^{t h} \\
\text { round's } \\
\text { input } \\
\text { mask }\end{array}$ & $\begin{array}{l}6^{t h} \\
\text { round's } \\
\text { input } \\
\text { mask }\end{array}$ & $\begin{array}{l}7^{t h} \\
\text { round's } \\
\text { input } \\
\text { mask }\end{array}$ & $\begin{array}{l}8^{t h} \\
\text { round's } \\
\text { input } \\
\text { mask }\end{array}$ & $\begin{array}{l}\text { Last } 0.5 \\
\text { round's } \\
\text { input } \\
\text { mask }\end{array}$ & $\begin{array}{l}\text { Cipher } \\
\text { text } \\
\text { mask }\end{array}$ & $\begin{array}{l}\text { \# of } \\
\text { free } \\
\text { key } \\
\text { bits }\end{array}$ \\
\hline 1 & 1100 & 0110 & 0110 & 1010 & 1100 & 0110 & 1010 & 1100 & 0110 & 0110 & 586 \\
\hline 2 & 1010 & 1100 & 0110 & 0110 & 1010 & 1100 & 0110 & 1010 & 1100 & 3100 & 586 \\
\hline 3 & 1010 & 1100 & 0110 & 0110 & 1010 & 1100 & 0110 & 1010 & 1100 & 1100 & 586 \\
\hline 4 & 0110 & 1010 & 1100 & 0110 & 0110 & 1010 & 1100 & 0110 & 1010 & 1010 & 586 \\
\hline 5 & 0110 & 1010 & 1100 & 0110 & 0110 & 1010 & 1100 & 0110 & 1010 & 3010 & 585 \\
\hline
\end{tabular}


Table 6. (continued)

\begin{tabular}{|c|c|c|c|c|c|c|c|c|c|c|c|}
\hline \# & \begin{tabular}{|l|}
$1^{s t}$ \\
round's \\
input \\
mask
\end{tabular} & \begin{tabular}{|l|} 
nd \\
round's \\
input \\
mask
\end{tabular} & \begin{tabular}{|l|}
$3^{r d}$ \\
round's \\
input \\
mask
\end{tabular} & \begin{tabular}{|l|}
$4^{t h}$ \\
round's \\
input \\
mask
\end{tabular} & \begin{tabular}{|l|}
$5^{t h}$ \\
round's \\
input \\
mask
\end{tabular} & \begin{tabular}{|l|}
$6^{\text {th }}$ \\
round's \\
input \\
mask
\end{tabular} & \begin{tabular}{|l|} 
th \\
round's \\
input \\
mask
\end{tabular} & \begin{tabular}{|l|}
$8^{t h}$ \\
round's \\
input \\
mask
\end{tabular} & $\begin{array}{l}\text { Last } 0.5 \\
\text { round's } \\
\text { input } \\
\text { mask }\end{array}$ & \begin{tabular}{|l|} 
Cipher \\
text \\
mask
\end{tabular} & $\begin{array}{l}\text { \# of } \\
\text { free } \\
\text { key } \\
\text { bits }\end{array}$ \\
\hline 6 & 0100 & 0001 & 0010 & 1011 & 1110 & 1101 & 0100 & 0001 & 0010 & 0010 & 579 \\
\hline 7 & 1001 & 0101 & 0011 & 1001 & 0101 & 0011 & 1001 & 0101 & 0011 & 0011 & 577 \\
\hline 8 & 0101 & 0011 & 1001 & 0101 & 0011 & 1001 & 0101 & 0011 & 1001 & 1001 & 577 \\
\hline 9 & 1001 & 0101 & 0011 & 1001 & 0101 & 0011 & 1001 & 0101 & 0011 & 0013 & 576 \\
\hline 10 & 0101 & 0011 & 1001 & 0101 & 0011 & 1001 & 0101 & 0011 & 1001 & 3001 & 576 \\
\hline 11 & 0101 & 0011 & 1001 & 0101 & 0011 & 1001 & 0101 & 0011 & 1001 & 1003 & 576 \\
\hline 12 & 0100 & 0001 & 0010 & 1011 & 1110 & 3101 & 0100 & 0001 & 0010 & 0010 & 576 \\
\hline 13 & 0101 & 0011 & 1001 & 0101 & 0011 & 1001 & 0101 & 0011 & 1001 & 3003 & 575 \\
\hline 14 & 1111 & 1111 & 1111 & 1111 & 1111 & 1111 & 1111 & 1111 & 1111 & 1111 & 562 \\
\hline 15 & 0011 & 1001 & 0101 & 0011 & 1001 & 0101 & 0011 & 1001 & 0101 & 0101 & 562 \\
\hline 16 & 1111 & 1111 & 1111 & 1111 & 1111 & 1111 & 1111 & 1111 & 1111 & 3111 & 561 \\
\hline 17 & 1111 & 1111 & 1111 & 1111 & 1111 & 1111 & 1111 & 1111 & 1111 & 1113 & 561 \\
\hline 18 & 0011 & 1001 & 0101 & 0011 & 1001 & 0101 & 0011 & 1001 & 0101 & 0103 & 561 \\
\hline 19 & 1111 & 1111 & 1111 & 1111 & 1111 & 1111 & 1111 & 1111 & 1111 & 3113 & 560 \\
\hline 20 & 1133 & 0100 & 0001 & 0010 & 3211 & 1133 & 0100 & 0001 & 0010 & 0010 & 557 \\
\hline 21 & 0100 & 0001 & 0010 & 3211 & 1133 & 0100 & 0001 & 0010 & 3211 & 1211 & 557 \\
\hline 22 & 0100 & 0001 & 0010 & 3211 & 1133 & 0100 & 0001 & 0010 & 3211 & 1213 & 556 \\
\hline 23 & 3311 & 1133 & 3311 & 1133 & 3311 & 1133 & 3311 & 1133 & 3311 & 1311 & 545 \\
\hline 24 & 1133 & 3311 & 1133 & 3311 & 1133 & 3311 & 1133 & 3311 & 1133 & 1131 & 545 \\
\hline 25 & 3311 & 1133 & 3311 & 1133 & 3311 & 1133 & 3311 & 1133 & 3311 & 1313 & 544 \\
\hline 26 & 1133 & 3311 & 1133 & 3311 & 1133 & 3311 & 1133 & 3311 & 1133 & 3133 & 544 \\
\hline 27 & 3211 & 1133 & 0100 & 0001 & 0010 & 3211 & 1133 & 0100 & 0001 & 0001 & 540 \\
\hline 28 & 3211 & 1133 & 0100 & 0001 & 0010 & 3211 & 1133 & 0100 & 0001 & 0003 & 539 \\
\hline 29 & 0001 & 0010 & 3211 & 1133 & 0100 & 0001 & 0010 & 3211 & 1133 & 1131 & 539 \\
\hline 30 & 0010 & 3211 & 1133 & 0100 & 0001 & 0010 & 3211 & 1133 & 0100 & 0100 & 538 \\
\hline 31 & 0001 & 0010 & 3211 & 1133 & 0100 & 0001 & 0010 & 3211 & 1133 & 3133 & 538 \\
\hline 32 & 1101 & 0100 & 0001 & 0010 & 1011 & 1110 & 1101 & 0100 & 0001 & 0001 & 534 \\
\hline 33 & 1110 & 1101 & 0100 & 0001 & 0010 & 1011 & 1110 & 1101 & 0100 & 0100 & 533 \\
\hline 34 & 1101 & 0100 & 0001 & 0010 & 1011 & 1110 & 1101 & 0100 & 0001 & 0003 & 533 \\
\hline 35 & 0010 & 1011 & 1110 & 1101 & 0100 & 0001 & 0010 & 1011 & 1110 & 1110 & 533 \\
\hline 36 & 0001 & 0010 & 1011 & 1110 & 1101 & 0100 & 0001 & 0010 & 1011 & 1011 & 533 \\
\hline 37 & 0010 & 1011 & 1110 & 1101 & 0100 & 0001 & 0010 & 1011 & 1110 & 3110 & 532 \\
\hline 38 & 0001 & 0010 & 1011 & 1110 & 1101 & 0100 & 0001 & 0010 & 1011 & 3011 & 532 \\
\hline 39 & 0001 & 0010 & 1011 & 1110 & 1101 & 0100 & 0001 & 0010 & 1011 & 1013 & 532 \\
\hline 40 & 3101 & 0100 & 0001 & 0010 & 1011 & 1110 & 3101 & 0100 & 0001 & 0001 & 531 \\
\hline 41 & 0001 & 0010 & 1011 & 1110 & 1101 & 0100 & 0001 & 0010 & 1011 & 3013 & 531 \\
\hline 42 & 3101 & 0100 & 0001 & 0010 & 1011 & 1110 & 3101 & 0100 & 0001 & 0003 & 530 \\
\hline 43 & 0010 & 1011 & 1110 & 3101 & 0100 & 0001 & 0010 & 1011 & 1110 & 1110 & 530 \\
\hline 44 & 0001 & 0010 & 1011 & 3110 & 1101 & 0100 & 0001 & 0010 & 1011 & 1011 & 530 \\
\hline 45 & 0001 & 0010 & 1011 & 1110 & 3101 & 0100 & 0001 & 0010 & 1011 & 1011 & 530 \\
\hline 46 & 0010 & 1011 & 1110 & 3101 & 0100 & 0001 & 0010 & 1011 & 1110 & 3110 & 529 \\
\hline 47 & 0001 & 0010 & 1011 & 3110 & 1101 & 0100 & 0001 & 0010 & 1011 & 3011 & 529 \\
\hline 48 & 0001 & 0010 & 1011 & 3110 & 1101 & 0100 & 0001 & 0010 & 1011 & 1013 & 529 \\
\hline 49 & 0001 & 0010 & 1011 & 1110 & 3101 & 0100 & 0001 & 0010 & 1011 & 3011 & 529 \\
\hline 50 & 0001 & 0010 & 1011 & 1110 & 3101 & 0100 & 0001 & 0010 & 1011 & 1013 & 529 \\
\hline
\end{tabular}




\section{References}

1. Chaves, R., Sousa, L.: Improving residue number system multiplication with more balanced moduli sets and enhanced modular arithmetic structures. IET Comput. Digital Tech. 1(5), 472-480 (2007)

2. Daemen, J., Govaerts, R., Vandewalle, J.: Weak keys for IDEA. In: Stinson, D.R. (ed.) CRYPTO 1993. LNCS, vol. 773, pp. 224-231. Springer, Heidelberg (1994). https://doi.org/10.1007/3-540-48329-2_20

3. Junod, P., Macchetti, M.: Revisiting the IDEA philosophy. In: Dunkelman, O. (ed.) FSE 2009. LNCS, vol. 5665, pp. 277-295. Springer, Heidelberg (2009). https://doi. org/10.1007/978-3-642-03317-9_17

4. Lai, X.: On the Design and Security of Block Cipher. ETH Series in Information Processing, vol. 1. Hartung-Gorre Verlag, Konstanz (1992)

5. Meier, W.: On the security of the IDEA block cipher. In: Helleseth, T. (ed.) EUROCRYPT 1993. LNCS, vol. 765, pp. 371-385. Springer, Heidelberg (1994). https:// doi.org/10.1007/3-540-48285-7_32

6. Modugu, R., Choi, M., Park, N.: A fast low-power modulo $2^{n}+1$ multiplier design. In: IEEE Instrumentation and Measurement Technology Conference, I2MTC 2009, pp. 951-956. IEEE (2009)

7. Nakahara Jr., J.: Personal communication, November 2004

8. Nakahara Jr., J.: Lai-Massey Cipher Designs: History. Design Criteria and Cryptanalysis. Springer, Cham (2018). https://doi.org/10.1007/978-3-319-68273-0

9. Nakahara Jr., J., Rijmen, V., Preneel, B., Vandewalle, J.: The MESH block ciphers. In: Chae, K.-J., Yung, M. (eds.) WISA 2003. LNCS, vol. 2908, pp. 458-473. Springer, Heidelberg (2004). https://doi.org/10.1007/978-3-540-24591-9_34

10. Nyberg, K.: On the construction of highly nonlinear permutations. In: Rueppel, R.A. (ed.) EUROCRYPT 1992. LNCS, vol. 658, pp. 92-98. Springer, Heidelberg (1993). https://doi.org/10.1007/3-540-47555-9_8

11. SageMath, the Sage Mathematics Software System (Version 6.7). The Sage Developers (2015). http://www.sagemath.org

12. Yıldırım, H.M.: Nonlinearity properties of the mixing operations of the block cipher IDEA. In: Johansson, T., Maitra, S. (eds.) INDOCRYPT 2003. LNCS, vol. 2904, pp. 68-81. Springer, Heidelberg (2003). https://doi.org/10.1007/978-3-540-24582$7 \_5$

13. Zhang, X.-M., Zheng, Y., Imai, H.: Duality of Boolean functions and its cryptographic significance. In: Han, Y., Okamoto, T., Qing, S. (eds.) ICICS 1997. LNCS, vol. 1334, pp. 159-169. Springer, Heidelberg (1997). https://doi.org/10. 1007/BFb0028472 